\title{
Estimating Atmospheric Boundary Layer Turbulence in the Marine Environment Using Lidar Systems with Applications for Offshore Wind Energy
}

by

Praneeth Gurumurthy

B.Tech.(Hons.), Indian Institute of Technology (2015)

M.Eng., Stevens Institute of Technology (2017)

Submitted to the Department of Earth, Atmospheric and Planetary

Sciences

in partial fulfillment of the requirements for the degree of

Master of Science in Physical Oceanography

at the

MASSACHUSETTS INSTITUTE OF TECHNOLOGY

and the

WOODS HOLE OCEANOGRAPHIC INSTITUTION

February 2021

(C) Massachusetts Institute of Technology and Woods Hole

Oceanographic Institution 2021. All rights reserved.

Author

Department of Earth, Atmospheric and Planetary Sciences January 15, 2021

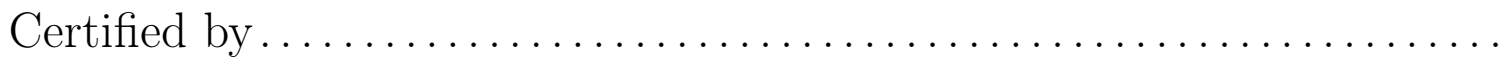

Anthony R. Kirincich

Associate Scientist

Woods Hole Oceanographic Institution

Thesis Supervisor

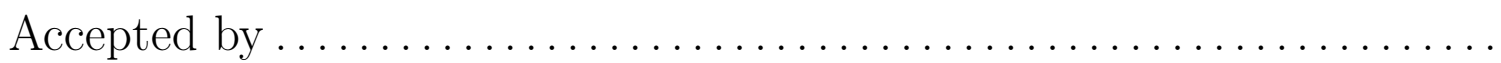

Glenn R. Flierl

Chair, Joint Committee for Physical Oceanography Massachusetts Institute of Technology/

Woods Hole Oceanographic Institution 


\title{
Estimating Atmospheric Boundary Layer Turbulence in the Marine Environment Using Lidar Systems with Applications for Offshore Wind Energy
}

by

Praneeth Gurumurthy

\author{
Submitted to the Department of Earth, Atmospheric and Planetary Sciences \\ on January 15, 2021, in partial fulfillment of the \\ requirements for the degree of \\ Master of Science in Physical Oceanography
}

\begin{abstract}
Estimating turbulence in the marine-atmospheric boundary layer is critical to many industrial, commercial and scientific fields, but of particular importance to the wind energy industry. Contributing to both the efficiency of energy extraction and the life-cycle cost of the turbine itself, turbulence in the atmospheric boundary layer is estimated within the wind energy industry as Turbulence Intensity (TI) and more recently by Turbulent Kinetic Energy (TKE). Traditional in-situ methods to measure turbulence are extremely difficult to deploy in the marine environment, resulting in a recent movement to and dependence on remote sensing methods. One type of remote sensing instrument, Doppler lidars, have shown to reliably estimate the wind speed and atmospheric turbulence while being cost effective and easily deployable, and hence are being increasingly utilized as a standard for wind energy assessments.

In this thesis, the ability of lidars to measure turbulence up to a height of $200 \mathrm{~m}$ above mean sea level in the marine-atmospheric boundary layer was tested using a 7-month data set spanning winter to early summer. Lidar-based TI and TKE were estimated by three methods using observations from a highly validated lidar system and compared under both convective and stable atmospheric stability conditions. Convective periods were found to have higher turbulence at all the heights compared to stable conditions, while mean wind speed and shear were higher during stable conditions. The study period was characterized by generally low turbulent conditions with high turbulence events occurring at timescales of a few days. Mean vertical profiles of TKE were non-uniformly distributed in height during low turbulent conditions. During highly turbulent events, TKE increased more strongly with height. The definition of TI- following the industry or meteorology conventions - had no real effect on the results, and differences between cup or sonic anemometers and lidar TI values were small except at low wind speeds. All the three lidar-based TKE methods tested corresponded closely to independent estimates, and differences between the methods were small relative to the temporal variability of TKE observed at the offshore site.
\end{abstract}


Thesis Supervisor: Anthony R. Kirincich

Title: Associate Scientist

Woods Hole Oceanographic Institution 


\section{Acknowledgments}

I would like to sincerely thank my advisor, Anthony Kirincich, for his continuous support, guidance, insight and everlasting patience. I would like to thank Jim Edson for providing the processed meteorological data used in this thesis. I would like to acknowledge the support from WHOI Academic Programs Office, Lea Fraser, Meg Tivey, Delia Oppo, Julia Westwater, and Christine Charette. I am grateful to Kris Kipp and Daisy Caban at MIT for their support. I would like to thank the Joint Committee for Physical Oceanography members Magdalena Andres, Jake Gebbie, Glenn Flierl, Claudia Cenedese and all the faculty members at MIT and WHOI for their support and guidance throughout my time in the Joint Program. My time in graduate school wouldn't have been fun without my friends and colleagues at MIT, in the Joint Program, and at pika. Lastly and importantly, I would like to thank my family for their support. 


\section{Contents}

1 Introduction $\quad 15$

2 Background $\quad 19$

3 Methodology 25

3.1 Study area and data availability . . . . . . . . . . . . 25

3.2 Instruments . . . . . . . . . . . . . . . . . . 26

3.3 Lidar methods for estimating turbulence . . . . . . . . . . . 27

3.3.1 Method 1: Eberhard method with 5 Beam (EB-5) . . . . . 29

3.3.2 Method 2- Doppler Beam Swinging- DBS . . . . . . . . . . 31

3.3.3 Method 3- DBS- correction . . . . . . . . . . . . . . . 32

3.4 Data Processing . . . . . . . . . . . . . . . . . 33

3.4 .1 Lidar . . . . . . . . . . . . . . . . . . 33

3.4.2 Sonic anemometer ................. 34

3.4.3 Cup anemometers and wind vane . . . . . . . . . . . . . 34

3.4 Atmospheric Stability . . . . . . . . . . . . 35

4 Results $\quad 37$

4.1 Mean conditions . . . . . . . . . . . . . . . . . 37

4.2 Turbulence Intensity $(\mathrm{TI})$ validation . . . . . . . . . . . . . . . . . . 39

4.3 Turbulent Kinetic Energy (TKE) validation . . . . . . . . . . . 41

4.4 Mean Turbulence Conditions . . . . . . . . . . . . . . . . . . 43

5 Discussions and Conclusion $\quad 47$ 
A Tables

B Figures 


\section{List of Figures}

B-1 ASIT met-tower. Windcube v2.0 (white box) is mounted on a platform $13 \mathrm{~m}$ above mean sea level. . . . . . . . . . . . . . . . . . . 60

B-2 Location of ASIT. Shaded area are lease areas for wind energy development . . . . . . . . . . . . . . . .

B-3 Top: 40-hour low pass mean wind speed for cup, sonic anemometers and Windcube v2. Middle: 40-hour low pass mean wind speed observed by Windcube v2.0 at 53, 120 and $180 \mathrm{~m}$ Bottom: 40-hour low pass mean wind direction observed by Windcube v2.0 at 53, 120 and 180 m. Wind direction is the direction from which the wind is coming from (meteorological convention), measured clockwise from North in degrees. 40-hour low pass filter is used to remove the diurnal effects. Sonic data is not available for the winter. . . . . . . . . . . .

B-4 Top to bottom: Distribution of wind direction, speed, and TI measured by cup anemometer and TKE estimated from sonic anemometer for Winter and Spring (before 01 March and afterwards). y-axis is the normalized frequency and wind direction is in meteorological convention as in (Fig: B-3). Sonic data is available for only 3 days in the winter and hence the distribution is not an accurate representation for full winter period. . . . . . . . . . . . . . . . . . 
B-5 Top to bottom: Distribution of wind direction, speed, and TI measured by cup anemometer and TKE estimated from sonic anemometer during times for wind coming from land (clockwise 270 to $90^{\circ}$ ) vs sea (clockwise 90 to $270^{\circ}$ ). $\mathrm{y}$-axis is the normalized frequency and wind direction is in meteorological convention as in (Fig: B-3). Sonic data is available only during spring. . . . . . . . . . . . . . . .

B-6 Mean wind speed profiles based on the height averaged wind speed distribution and stability conditions. The distribution is divided into three quantiles with equal number of data points in each quantiles (33.33 percentile bins). Error bars are standard errors of mean estimated by method described in Ch:3.4.1 . . . . . . . . . . . . .

B-7 Turbulence intensity distribution and comparison to mean wind speed measured by cup anemometer. . . . . . . . . . . . . . . . 66

B-8 Comparison of TI using Meteorological (left panels) and Industry convention (right) to calculate TI for WindCube v2.0 lidar at $53 \mathrm{~m}$ amsl (top) and sonic anemometer at $20 \mathrm{~m}$ amsl (bottom) with cup anemometer at $26 \mathrm{~m}$ amsl. The black line shows the slope 1 line and red line shows the linear regression fit, slope and the Pearson correlation coefficient are displayed on the panel. The red markers with error bars are bin averaged TI. . . . . . . . . . . . . . . .

B-9 Comparison of TI for the full period for: DBS, DBS corrected and EB-5 methods for WindCube v2.0 lidar at 53m and sonic anemometer at $20 \mathrm{~m}$ (Sonic data available only from late February onwards) with cup anemometer at $26 \mathrm{~m}$ amsl. Statistics displayed on the figure are same as in (Fig: B-8). . . . . . . . . . . . . . . . . . . . 68

B-10 Comparison of TI during convective (left) and stable (right) for (top to bottom): DBS method, DBS corrected method, EB-5 method and sonic anemometer (Sonic data available only from late February onwards) with cup anemometer at $26 \mathrm{~m}$ amsl. Statistics displayed on the figure are same as in (Fig: B-8) . . . . . . . . . . . . . . . 
B-11 TKE comparison of (top to bottom): DBS, DBS corrected and EB-5 method for WindCube v2.0 lidar at $53 \mathrm{~m}$ amsl with sonic anemometer at $20 \mathrm{~m}$ amsl from late February onwards. The black line shows the slope 1 line and red line shows the linear regression line, slope. The Pearson correlation coefficient are displayed on the panel. The red markers with error bars are bin averaged TKE. Error bars are standard error of mean computed as described in Sec. 3.4.1 . . . . . . . . . . .

B-12 log-TKE comparison of the three methods for lidar at $53 \mathrm{~m}$ amsl with TKE measured by sonic anemometer at $20 \mathrm{~m}$ amsl from late February onwards. Statistics displayed on figure are same as in (Fig: B-11).The slope and Pearson correlation coefficient for log-TKE linear regression fit is displayed. . . . . . . . . . . . . . . . . .

B-13 TKE comparison during convecive (left) and stable (right) of (top to bottom): DBS, DBS corrected and EB-5 method for WindCube v2.0 lidar at $53 \mathrm{~m}$ amsl with sonic anemometer at $20 \mathrm{~m}$ amsl from late February onwards. Statistics displayed on figure are same as in B-11 .

B-14 log-TKE comparison during convecive (left) and stable (right) of (top to bottom): DBS, DBS corrected and EB-5 method for WindCube v2.0 lidar at $53 \mathrm{~m}$ amsl with sonic anemometer at $20 \mathrm{~m}$ amsl from late February onwards. Statistics displayed on figure are same as in (Fig: B-11). The slope and Pearson correlation coefficient for log-TKE linear regression fit is displayed. . . . . . . . . . . . . .

B-15 40-hour low pass filtered TI time series for DBS and EB-5 method for WindCube v2.0 lidar at $53 \mathrm{~m}$ and $180 \mathrm{~m}$ amsl. The Pearson correlation coefficient between time series at the two heights is displayed on the panel. . . . . . . . . . . . . . . . . . . . . 74

B-16 Mean TI profile for DBS (left) and EB-5 (right) during convective and stable conditions and for the full study period. . . . . . . . . . . 
B-17 40-hour low pass filtered TKE time series for DBS and EB-5 method for WindCube v2.0 lidar at $53 \mathrm{~m}$ and $180 \mathrm{~m}$ amsl. The Pearson correlation coefficient between time series at the two heights is displayed on the

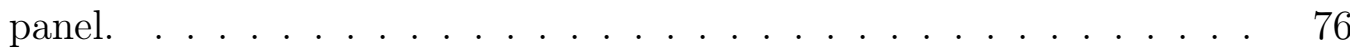

B-18 Mean TKE profile for DBS (left) and EB-5 (right) during convective and stable conditions and for the full study period. . . . . . . . . . 77

B-19 Top: Mean TKE profile for four bins (shown in bottom panel) for stable (grey) and convective (black). Bottom: Height averaged TKE distribution using DBS method for stable (grey) and convective (black). The bins are divided as $(0,0.4)(0.4,1)(1,2)(>2)$ and shown with vertical lines. The standard error of mean is estimated by method described in Sec.3.4.1. . . . . . . . . . . . . . 78

B-20 Mean TKE profile for the first bin (TKE 0 to $0.4 \mathrm{~m}^{2} / \mathrm{s}^{2}$ ) in Figure: B-19 for DBS (triangles) and EB-5 (cross) during convective (black) and stable (grey) conditions. The standard error of mean is estimated by method described in Sec.3.4.1. . . . . . . . . . . . . 


\section{List of Tables}

A.1 Percentage of good data for sonic anemometer, cup anemometer and Windcube v2.0 lidar at heights shown above mean seal level. . . . . . 56

A.2 Classification of stability- percentage of stable and convective conditions during the study period . . . . . . . . . . . . 57 


\section{Chapter 1}

\section{Introduction}

Characterizing atmospheric turbulence is critically important in many industrial and commercial applications; including modelling forest fires [1], understanding aircraft wakes at airports [2], and for designing wind farms. For wind energy applications specifically, understanding turbulence is important because the turbulence dissipation rate determines how long the wakes from a turbine will persist, i.e. the downstream extent of the wakes $[3,4,5]$, and thus the effect of each turbine on neighboring turbines [6]. Turbulence determines the wind speed on the rotor disk $[7,8]$ and the power output of the turbine itself $[9,10]$. Turbulence also impacts the load on the wind turbine structure $[11,12,13]$ and impacts the lifetime of the turbine $[14,15]$. In the design process, the magnitude and variability of turbulence are critical parameters in determining the location, size and arrangement of the wind farms [16]. During wind farm operations, understanding the turbulence characteristics of the flow field is important for forecasting power generated from wind turbines [17] as well as planning for turbine maintenance operations.

To estimate wind speed and turbulence spanning the wind turbine rotor, cup anemometers and sonic anemometers mounted on tall meteorological masts (met masts) have been traditionally used [18]. Low cost cup anemometers have been known to be less precise due to poorer response times and over-speeding [19, 20]. More expensive sonic anemometers, in contrast, have faster sampling rates and measure variance more precisely. However, both cup and sonic anemometers must be 
mounted on a fixed structure at the measurement height, making them economically infeasible for many studies, particularly in the offshore environment. With increasing turbine sizes, larger rotor diameters, and expansion into deeper offshore water depths, deploying met masts for characterizing the wind resource is not economically feasible. Remote sensing devices [21] like lidar (light detection and ranging) [22, 23], sodar (sound detection and ranging) [24] and floating lidars (lidars mounted on buoys, also called flidars) [25], on the other hand, provide alternative solutions by remotely measuring horizontal mean wind velocities at multiple heights reaching up to the upper tip of the rotor blade.

In the wind energy industry, turbulence in the atmospheric boundary layer has generally been characterized by turbulence intensity (TI). TI is defined as the standard deviation of horizontal wind speed normalized by mean of horizontal wind speed over the sampling period (generally 10 minutes). The industry standard for this parameter is specific to what can be measured by cup anemometers mounted on met masts. Previous studies have shown that TI does not take into account the amount of turbulence due to vertical velocity fluctuations [19] and therefore might not accurately represent the turbulence conditions present at high wind speeds and in convective conditions. Other turbulence parameters, such as turbulent kinetic energy (TKE), defined as the weighted sum of variances of all three components of velocity, have proven to be better because they account for fluctuations in all three directions [18].

TKE can be measured by remote sensing devices mentioned earlier or by sonic anemometers, but not cup anemometers. While generally giving optimal estimates of TKE, sonic anemometers have the same disadvantages as cup anemometers, namely the inability to measure at multiple heights with one instrument and the need to deploy a met mast to measure across the potential turbine height. As a result they are economically infeasible for a holistic wind resource assessment. Ground, tower, or buoy-mounted vertically profiling lidars can offer all the advantages of a series of cup or sonic anemometers on a met mast, while also measuring a reasonably good estimate of turbulence and mean wind speed. Over the last decade, several studies 
have developed techniques and configurations to estimate turbulence statistics from lidar [26] and each of these methods have some advantages and disadvantages.

This thesis compares the performance of a number of lidar-based turbulence estimation methods in the offshore environment using both the commonly used turbulent statistics- TKE and TI. In general, lidar-based TKE and TI methods are shown to be reliable indicators of turbulent fluctuations when compared to industry standard in-situ sensors - cup and sonic anemometers. These estimates are used to characterize changes in turbulence statistics within the marine-atmospheric boundary layer at an offshore metocean tower (Fig: B-1) over a range of seasonal and atmospheric stability conditions. The tower is located south of Martha's Vineyard, Massachusetts along the U.S. outer continental shelf, an area of intensive interest for offshore wind energy development in the U.S. (Fig: B-2). Examined during a seven-month period spanning winter to early summer, the differences between the lidar-based turbulence estimation methods were found to be not as great as the seasonal and stability related changes in the turbulence characteristics of the marine atmospheric boundary layer.

The thesis begins with a brief background on turbulence statistics commonly used in the wind energy industry, and the methods used to estimate these quantities from vertically profiling lidars. This is followed by a brief description of the study site, the instruments used, details on the formulation of lidar-based turbulence statistics and the data processing methods employed here. The results are presented for validating the lidar observations of TI and TKE with in-situ observations from cup and sonic anemometer, followed by results on relative differences between lidar-based observations for a series of turbulence estimation methods. An analysis of the vertical structure of turbulence present during the study period is then presented, and the thesis concludes with a discussion of the results and their implications for the use of lidars as well as the conditions at the site for the wind energy industry. 


\section{Chapter 2}

\section{Background}

Turbulence in the atmosphere can be statistically quantified in many ways [27]. In the wind energy industry, it is common for turbulence to be quantified by either Turbulence Intensity (TI) or Turbulent Kinetic Energy (TKE), which are two different statistical measurements. The IEC 61400 standard [20] recommends TI to estimate the turbulence and assess the wind resource at a site. TKE measures the turbulence as a sum of all sources of turbulent fluctuations whereas TI is a measure of only horizontal turbulent fluctuations normalized by the mean horizontal wind speed. These turbulence parameters can be measured by a range of instruments using different techniques. In the wind energy industry, cup anemometers are the most utilized sensors to measure wind speed, however sonic anemometers and lidars are being increasingly deployed. Sonic anemometers use difference in time of flights of ultra sonic sound in a very small (centimeter scale) volume to infer the wind velocities in three directions. In contrast cup anemometers are mechanical sensors which output the speed of sound based on the speed of the cups accelerated by the horizontal wind speed. While cup anemometers can only measure turbulence in the horizontal plane, lidar and sonic anemometers have the potential to measure turbulence in all three directions. Moreover, to measure turbulence at heights spanning the wind turbine rotor, tall met masts must be deployed to mount multiple cup or sonic anemometers, adding significant cost especially in offshore sites. Hence, although the cup anemometers are relatively inexpensive, the cost to perform a wind resource assessment using cup and 
sonic anemometers is significantly higher offshore compared to lidars.

Depending on the volume scanned relative to the lidar position, lidars can be vertical plane scanning lidars- which can adjust the elevation angle (Range Height Indicator- RHI), or profiling lidars -(vertical staring and scanning) which measure the volume directly above the lidar [26]. Furthermore, depending on the probing signal, lidars can be classified as continuous wave or pulsed lidars (finite pulse length called probe length). Both continuous wave (CW) and pulsed lidars have advantages and disadvantages in their performance, data return, and uncertainty [28, 29]. Both sampling types use the Doppler shift of the reflected signal to estimate the velocity of aerosol particles within the sampling volume. In CW lidars, a continuous signal is transmitted and the azimuth angle is changed to form a sampling volume of a cone extending from the lidar. The measurements at different heights are made by focusing the intensity of the lidar beam at a particular height and using computational techniques to filter out reflections from other heights. Therefore, a circle is scanned at each height. In contrast, pulsed lidars send a single pulse, obtain the reflections from all the heights, range-gating the signal into separate vertical measurement volumes. This is done at a finite number of azimuth angles for each pulse, resulting in discrete sampling volumes from multiple heights. The data processing technique for the conical scanning CW lidars is called Velocity Azimuth Display (VAD) technique [26] while that for the pulsed lidars is called Doppler Beam Swinging (DBS) technique [30].

The wind energy industry has historically relied on cup anemometer observations to measure horizontal wind speeds for resource characterization and power production estimates. To estimate the turbulence, TI is defined in the wind energy industry as the standard deviation of horizontal wind speed divided by mean wind speed measured by cup anemometer. This definition does not take the vertical velocity fluctuations into account. On the other hand, TI can also be estimated by sonic anemometers and lidars, both of which measure wind velocity in in all three directions (x,y,z; i.e. two horizontal directions and one vertical direction). There are several methods proposed $[19,31,32,33]$ to compute TI from the three velocity components measured by lidars or sonic anemometers to compare to the TI estimated from cup anemometers, each 
of which gives a different estimate of the turbulence present. Two such methods are discussed and compared in this thesis.

Despite their popularity, cup anemometers have a number of known issues that can degrade their ability to accurately assess the turbulence present. Cup anemometers have a potential bias in that they tend to respond faster to increases in velocity than to decreases in velocity, leading to an effect called 'over-speeding' [34]. TI measured by cup anemometer is also less reliable at very low wind speeds (less than $1 \mathrm{~m} / \mathrm{s}$ ) due to both the performance of cup anemometers at low winds and the normalization by the horizontal mean wind speed employed by TI [35]. Thus, when turbulent fluctuations are estimated by cup anemometers, they can result in a potentially biased estimate of the turbulence levels present.

TKE can be measured by both lidars and sonic anemometers, yet both sensor types measure this critical parameter differently. Sonic anemometers measure velocity fluctuations in all three directions ( $\mathrm{x}, \mathrm{y}$, and $\mathrm{z}$; two horizontal and one vertical directions) at a centimeter-scale sample volume using very high frequency $(\sim 20 \mathrm{~Hz})$ sampling. Remotely sensed, lidar-based estimates of TKE are notably different due to the sampling method used to measure the radial velocities and infer the horizontal and vertical wind velocities. That lidars measure the wind speed by using radial beams that diverge from the instrument means that any estimate of wind speed entails varying amount of spatial averaging depending on the method used and the height of the measurement. Hence, lidar-based TKE estimates are not a single-point measurement but a volume-averaged estimate. The measuring volume, defined as the area of scanning circle times the range cell length, increases with the height, causing the measurement to be made over an effectively larger volume at higher heights. The integral length scale of turbulence (here on referred to as turbulent length scale) - the size of the dominant energy-carrying eddies [36]- are likely to vary with height [37], meaning the impact of this filtering effect depends on the ratio of the local turbulent length scale to measuring volume size [38]. Additionally, due to lidar sampling methods, the sampling frequency is commonly much lower than that of the sonic anemometers. Both the sampling frequency and the sample volume determines the turbulent length 
scales that can be measured by the instrument. For example, turbulent length scales that are smaller than the instruments sampling volume will be under resolved by the lidar system.

Use of remote sensing instruments to measure the turbulence characteristics of the atmospheric boundary layer has been advancing for decades, since the first observations from Doppler Radars were utilized during the 1970s. Eberhard et al [39] was one the earliest studies which demonstrated the use of Doppler lidar to measure turbulence properties in the atmospheric boundary layer. Since then a number of techniques have been developed to measure turbulence parameters by lidars [29, 4, 40, 39, 41]. Broadly, they can be classified as (1) estimating the turbulence from earth coordinate velocities calculated from the directly measured radial velocities via a geometric transformation (Doppler Beam Swinging- DBS) [30, 42, 43] or (2) estimating turbulence directly from the radial (or along-beam) velocities of the individual beams (referred as here as the EB method) [39, 41].

In both the methods, the principal measurement is the radial velocity (which is estimated by the Doppler shift of the laser signal received). However, given the geometric transformation into earth coordinate velocities used by the DBS method, each method makes different assumptions about the characteristics of turbulence and how they are observed by the instrument.

Using the DBS method to estimate turbulence fluctuations assumes that both the mean flow field and the turbulent flow field are homogeneous [44] in the sample volume (which increases with height). In effect, this method assumes the instrument measures the same eddy at the same time across all beams. As the beams are generally not sampled at the same time, this method also assumes that the turbulence is 'frozen' over the sampling timescale. This leads to significant averaging of the turbulence scales smaller than that of the sampling volume and time scales smaller than that of sampling period of the lidar, as mentioned above.

In the EB method, by using radial velocities directly to estimate turbulence, only the statistics of turbulence are assumed to be homogeneous in the sampling volume but not the flow field. This difference should act to reduce some of the effects of the 
spatial averaging on the estimated TKE using the EB methodology. Hence, using the direct estimates from the radial velocities themselves, rather than transformed velocity variances, circumvents both the frozen turbulence assumption as well as the assumption of horizontal homogeneity. Another way to circumvent the horizontal homogeneity assumption is to use multiple lidars measuring at the same point [45]. However, this is rarely economically feasible for most studies. In addition to the 'spatial' (measuring volume) averaging, both these methods also have error due to averaging within the probe length itself (the sample volume illuminated by the laser). This probe length is constant for pulsed lidars (around 20m for the WindCube v2) while it increases with height for CW lidars. The probe volume averaging is insignificant because the turbulence length scales in the atmospheric boundary layer are usually larger [37] than the probe volume. Finally, in addition to the sampling errors, there are systematic errors induced by the instrument noise [46, 23]. For pulsed lidar systems, Sathe et al [26] recently introduced a six beam approach, based on EB method, which was able to obtain all six turbulent components of the symmetric Reynolds stress tensor from the radial velocity fluctuations. This technique was also examined by Bonin et al [47], who's results suggested that lidars are not skilled at estimating the velocity co-variances (off-diagonal elements in the stress tensor, i.e. the Reynolds stresses).

The standard deviation used in TI calculation can be obtained by root mean square of individual velocity variances ( in case of sonic and lidar) or by standard deviation of the horizontal wind speed (for cup anemometer). The former method is used by meteorologists (and referred here as meteorological convention) while the latter is used by the wind energy industry (referred here as industry convention) [19]. On the other hand, by including velocity fluctuations in all three directions, TKE gives a better understanding for the origin of turbulence while TI does not [18].

This study focuses on the turbulent quantities TI and TKE which require only the velocity variances- i.e. the diagonal terms in the Reynolds stress tensor. The turbulent quantities were estimated for a cup anemometer, sonic anemometer and a WindCube v2.0 lidar. These instruments were deployed on a $23 \mathrm{~m}$ tall offshore met 
tower, located $10 \mathrm{NM}$ from multiple offshore wind energy development lease areas within the U.S. East Coast outer continental shelf as part of a long term metocean monitoring campaign. By leveraging this unique data set, this work aims to understand the potential differences between commonly estimated turbulence statistics at the site as well as make an assessment of the various methods to extract lidar-based turbulence observations. We use a similar approach as that introduced by Eberhard et al [39], but by using four slant beams and a vertical beam we solve for the TI and TKE without needing velocity observations at two elevation angles. Previously, Newman et al. [42] used a 5-beam Windcube v2.0 lidar to estimate TKE with the DBS technique and compared the results to the six beam approach [41] described above. The DBS method was found to overestimate the $\mathrm{u}$ and $\mathrm{v}$ variances compared to sonic anemometers, due in part to variance contamination in DBS arising as a result of differences in instantaneous velocities at different beams. Newman et al. [42] then added applied a correction to the DBS variance estimate to attempt to account for this contamination issue by approximating the auto-correlation between the instantaneous velocities using two sonic anemometers separated by $11.5 \mathrm{~m}$. As this was found to help reduce the overestimation of variance by $20 \%$, a similar correction technique is evaluated here as well. 


\section{Chapter 3}

\section{Methodology}

\subsection{Study area and data availability}

This study used data obtained from a long term Met-ocean monitoring site located at the Woods Hole Oceanographic Institution's Air-Sea Interaction Tower (ASIT: Fig. B-1), a fixed tower located 3-Km offshore of the island of Martha's Vineyard, Massachusetts (Fig: B-2). The ASIT is in $17 \mathrm{~m}$ of water and approximately $10 \mathrm{NM}$ north of the the Rhode Island and Massachusetts wind energy lease areas (Fig. B-2). Operational since 2003, the 23-m tall structure serves as a platform for a suite of met-ocean sensors maintained for both basic and applied research on the ocean and atmosphere [48], including anemometers; air temperature, air pressure, and relative humidity; sea surface temperature and salinity; and surface gravity waves. Larger instruments like the Leosphere Windcube v2.0 lidar system used here can also be deployed at the ASIT, using a platform located 13m above mean sea level (amsl) (Fig: B-1). The ASIT is a unique offshore platform in the U.S., exposed to predominantly open ocean wind and wave conditions from the South, but land-affected during times when the winds are from the North.

In addition to the core sensors maintained by WHOI for long-term monitoring, a set of wind-energy specific sensors were deployed on the ASIT starting in 2016, as part of a met-ocean initiative funded by Massachusetts Clean Energy Center. As part of this initiative, a Leosphere Windcube v2.0 lidar system was installed in the 
fall of 2016, serviced in 2019, and re-validated against a tall meteorological tower in the summer of 2019. More information about the initiative can be found in Kirincich 2020 [48]. Details about the initiative-deployed sensors are given below.

\subsection{Instruments}

We use data from the following instruments in this study:

- Lidar- The Leosphere Windcube v2.0 lidar is a vertically profiling lidar which uses the Doppler Beam Swinging (DBS) technique to measure wind velocities at 11 heights up to $200 \mathrm{~m}$ at a sampling frequency of $1 \mathrm{~Hz}$. The Windcube v2.0 has four fixed beam locations, measuring line of sight velocity at elevation angles of $62^{\circ}$ above the horizon, that are each $90^{\circ}$ apart in azimuth. A fifth beam points vertically upwards. Each beam is sampled sequentially for approximately 1 second, therefore a complete cycle of measurements takes up to 5 seconds to complete. The raw data returned from the lidar include radial wind speed, CNR (Carrier-to-Noise ratio), and radial wind speed dispersion at 11 heights $(53,60,80,90,100,110,120,140,160,180$, and $200 \mathrm{~m}$ amsl), spanning the rotor area of a typical wind turbine. In addition to the above quantities, the Windcube v2.0 uses the DBS method to compute an independent estimate of earth coordinate horizontal velocities and wind direction once every sample cycle (around 5 seconds), but displays a trailing estimate every second. The lidar data used in this study spans the period from October 282019 to June 19 2020, encompassing the deployment period starting right after the tall-tower validation of the lidar and ending when an optical chain failure of the lidar required its removal for servicing.

- Cup anemometer: A cup anemometer- P2546c-OPR, located at $26 \mathrm{~m}$ amsl measured wind speed while the wind direction was measured by rNRG 200P wind vane at $23 \mathrm{~m}$ amsl, sampled at $1 \mathrm{~Hz}$. This data is available from Nov 22 2019 to June 202020. 
- Sonic anemometer: The wind velocity data was measured by two sonic anemometers (WindMasters until 28 May 2020 and R3's thereafter) at $20 \mathrm{~Hz}$. The raw data returned from sonic anemometers include wind velocities in the three cardinal directions, wind direction and air temperature. These instruments were placed $6 \mathrm{~m}$ apart and located at $20 \mathrm{~m}$ amsl. The data is available from February 26 to June 122020.

- Raw Meteorological data: The air temperature data was measured by Vaisala HMP45A-P mounted at $20 \mathrm{~m}$ amsl and the water temperature was measured by SBE $37 \mathrm{CT}$ at $4 \mathrm{~m}$ below mean sea level. The data is available at $1 \mathrm{~Hz}$ from February 26 to June 042020.

- Processed Meteorological data: A processed data set consisting of air temperature, pressure, relative humidity, sea surface temperature, friction velocity and buoyancy flux averaged to 20-minute intervals was also available at ASIT via a separate experiment conducted at the tower. Obtained directly from the PI Jim Edson, this data is used to estimate the stability conditions by calculating Obukhov length at 20-minute intervals. This data is available from October 16 2019 to February 122020.

\subsection{Lidar methods for estimating turbulence}

Two turbulence parameters were estimated via the lidar and sonic or cup anemometers: turbulence intensity (TI) and turbulent kinetic energy (TKE). In wind energy industry (ind) TI is defined as:

$$
T I_{i n d}=\frac{\sigma_{U}}{U}
$$

where $\mathrm{U}$ is the mean of the horizontal wind speed and $\sigma_{U}$ is the standard deviation of the horizontal wind speed over a 10-minute interval. However, in meteorology (met), TI is defined by the American Meteorological Society (AMS) [49] and in previous 
studies $[32,31]$ as the root mean square of velocity fluctuations, which gives:

$$
T I_{m e t}=\frac{\sqrt{\frac{\sigma_{u}^{2}+\sigma_{v}^{2}}{2}}}{U}
$$

where $\sigma$ represents the standard deviation of the velocity components $(u, v)$, and $\mathrm{U}$ is the mean of the horizontal wind speed. The industry and meteorological convention can result in different standard deviation as squaring the velocity components individually and adding them, rather than the combined metric of the wind speed, as a scalar, leads to extra terms in the wind speed computed TI that are not measured by cup anemometers. But we show in the results (Fig: B-8) that the two conventions, result in nearly equal estimates of standard deviation and consequently TI. Note that the meteorological definition used here is different from the meteorological definition used by Wharton and Lundquist [19], where the square root of the sum of squares was used instead of root mean square of the velocity fluctuations. The Wharton and Lundquist definition will therefore result in a standard deviation and consequently $\mathrm{TI}$, that is $\sqrt{2}$ times greater than the AMS definition used in this study.

TKE is defined as:

$$
T K E=\frac{1}{2}\left[\sigma_{u}^{2}+\sigma_{v}^{2}+\sigma_{w}^{2}\right]
$$

While estimates of TI or TKE are straightforward to make from sonic or cup anemometers, three different methods for estimating turbulence parameters from vertically profiling lidars are presented and discussed. First, a variation of the original Velocity Azimuth Display (VAD) technique [39] which is applicable to a WindCube v2.0 lidar with 5 beams, here onwards called the EB-5 method, is presented. Second, we discuss the commonly used approach called Doppler Beam Swinging, here onwards referred to as the DBS technique. While DBS is inherently used by the WindCube v2.0 to calculate earth coordinate velocities from radial velocity, it also serves as an approach to calculate the turbulence parameters by using equations [3.1c] and [3.1b]. A third method is also tested which uses corrections to the DBS method, here onwards referred as DBS-corr method, to correct for the distance between measuring 
beams and accounting for measuring different samples at different times by the individual beams. This method was first proposed by Newman et al [42] using correlation between the velocities at the beam positions.

\subsubsection{Method 1: Eberhard method with 5 Beam (EB-5)}

Eberhard et al [39] used a short-pulse $\mathrm{C}_{2}$ Doppler lidar to measure vertical profiles of turbulence parameters using the VAD scanning strategy. Using partial Fourier decomposition, they relate weighted sum of horizontal and vertical velocity variance to the average of radial velocity variances. We use a similar method, but instead of using multiple radial velocities across a scanning cone through VAD, we average across the four slant beams of the WindCube v2.0. In addition to the four slant beams, the WindCube's vertical pointing beam is used to measure the vertical velocity variance directly.

The measured radial velocity is given as:

$$
\hat{V}_{r}=V_{r}+\epsilon_{I}
$$

where: $\hat{V}_{r}$ is the measured radial velocity, $V_{r}$ is the true radial velocity, and $\epsilon$

represents the instrument error; assumed to be independent of $\hat{V}_{r}$, the position of the beam, and time. Following Eberhard's derivation [39], the ensemble average radial velocity variance, $v_{r}^{2}$, is written as:

$$
\left\langle v_{r}^{2}(R, \theta, \phi)\right\rangle=\left\langle\left[\hat{V}_{r}(R, \theta, \phi, t)-\left\langle\hat{V}_{r}(R, \theta, \phi, t)\right\rangle\right]^{2}\right\rangle
$$

where: $<>$ means ensemble average, $R, \theta$, and $\phi$ are the range, the azimuth angle, and the elevation angle (constant) respectively. Using geometric transformation, the radial velocity, as a function of range, azimuth, and elevation angle, can be converted to earth coordinate velocities $u, v$, and $w$. Substituting the transformation into 
equation (3.3a) gives:

$$
\begin{array}{r}
\left.\left\langle v_{r}^{2}(R, \theta, \phi)\right\rangle=\frac{\cos ^{2} \phi}{2}\left[\left\langle u^{2}\right\rangle+\left\langle v^{2}\right\rangle+2 \tan ^{2} \phi\left\langle w^{2}\right\rangle\right]+\left\langle\epsilon_{I}^{2}\right\rangle\right] \\
+\sin 2 \phi\langle u w\rangle \cos \theta-\sin 2 \phi\langle v w\rangle \sin \theta \\
+\frac{\cos ^{2} \phi}{2}\left[\left\langle u^{2}\right\rangle-\left\langle v^{2}\right\rangle\right] \cos 2 \theta-\cos ^{2} \phi\langle u v\rangle \sin 2 \theta
\end{array}
$$

A partial Fourier decomposition of equation (3.3b) with an assumption of horizontally homogeneous turbulent statistics gives:

$$
\left\langle u^{2}\right\rangle+\left\langle v^{2}\right\rangle+2 \tan ^{2} \phi\left\langle w^{2}\right\rangle+\frac{2}{\cos ^{2} \phi}\left\langle\epsilon_{I}^{2}\right\rangle=\frac{1}{\pi \cos ^{2} \phi} \int_{0}^{2 \pi}\left\langle v_{r}^{2}\right\rangle d \theta .
$$

For a pulsed lidar, the integral can be summed over the finite number of beams, four in case of WindCube v2.0. Re-writing the integral as a summation of discrete radial velocity variances and then taking their average gives:

$$
\left\langle u^{2}\right\rangle+\left\langle v^{2}\right\rangle+2 \tan ^{2} \phi\left\langle w^{2}\right\rangle+\frac{2}{\cos ^{2} \phi}\left\langle\epsilon_{I}^{2}\right\rangle=\frac{2}{\cos ^{2} \phi}\left\langle\left\langle v_{r}^{2}\right\rangle\right\rangle_{\theta},
$$

where \langle\rangle$_{\theta}$ represents an average over the four beams. Using the definitions of TI and TKE (Eqns: $3.1 \mathrm{~b}$ and $3.1 \mathrm{c}$ ) and rewriting the $\left\langle w^{2}\right\rangle$ term gives:

$$
T I_{E B-5}=\frac{\sqrt{\frac{1}{\cos ^{2} \phi}}\left\langle\left\langle v_{m}^{2}\right\rangle\right\rangle_{\theta}-2 \tan ^{2} \phi\left\langle w^{2}\right\rangle}{U}
$$

where $v_{m}^{2}$ is the mixed variance which combines the true variance $v_{r}^{2}$ and the instrument noise $\epsilon_{I}^{2}$ and $\mathrm{U}$ is the mean horizontal wind speed estimated by the lidar.

$$
T K E_{E B-5}=\frac{1}{2} \sqrt{\frac{2}{\cos ^{2} \phi}\left\langle\left\langle v_{m}^{2}\right\rangle\right\rangle_{\theta}+\left(1-2 \tan ^{2} \phi\right)\left\langle w^{2}\right\rangle}
$$

Note that this formulation uses the earth coordinate mean horizontal velocity in equation (3.5a) which is a standard in the wind energy industry. Many studies have shown lidars to estimate horizontal wind speeds with appreciable accuracy but not the velocity variances because of the assumptions stated in Ch. 2. During some bursts, 
the $T K E_{E B-5}$ resulted in a negative TKE due to high variance in the vertical velocity. This is symptomatic of errors in the estimate and without a physical interpretation. As a result, we remove negative values (around $7 \%$ of the $T K E_{E B-5}$ values) from analysis, following Newman et al [42] and Bonin et al [47].

\subsubsection{Method 2- Doppler Beam Swinging- DBS}

Doppler Beam Swinging (DBS) has been traditionally used to obtain earth coordinate velocities from radial velocities using geometric transformation. The radial velocity $v_{r}$ of each beam can be expressed using earth coordinate velocities $u, v, w$, and the elevation angle $\phi$ at the four slant beams (1 to 4 ) as:

$$
\begin{gathered}
v_{r 1}=v_{1} \cos \phi+w_{1} \sin \phi \\
v_{r 2}=u_{2} \cos \phi+w_{2} \sin \phi \\
v_{r 3}=-v_{3} \cos \phi+w_{3} \sin \phi \\
v_{r 4}=-u_{4} \cos \phi+w_{4} \sin \phi
\end{gathered}
$$

Solving for the earth coordinate velocities $u, v$, and $w$ by assuming horizontally homogeneous flow gives:

$$
\begin{aligned}
& \bar{u}=\frac{\overline{v_{r 2}}-\overline{v_{r 4}}}{2 \cos \phi} \\
& \bar{v}=\frac{\overline{v_{r 1}}-\overline{v_{r 3}}}{2 \cos \phi}
\end{aligned}
$$

For WindCube v2.0 the vertical velocity can be estimated directly from the fifth vertical beam as:

$$
\bar{w}=\overline{v_{r 5}}
$$

The TI and TKE can be computed from equations (3.1b) and (3.1c) by calculating the variances of $u, v$, and $w$ obtained from equations (3.7). Additionally, $T I_{\text {ind }}$ (the industry convention TI) was estimated by computing the horizontal wind speed from $u$ and $v$ using equation (3.1a). 


\subsubsection{Method 3- DBS- correction}

In order to take into account the in-homogeneity of the turbulence fluctuations across the scanning circle, Newman et al [42] used a correction to the DBS method (Sec: 3.3.2), based on the correlations between individual velocities at each slant beam (i.e. $u_{1}$ and $w_{1}$ are observed at beam 1 , while $u_{3}$ and $w_{3}$ are observed at the opposing beam 3). Following Newman et al [42], and assuming that the time-averaged correlations between the velocity components observed at a beam were equal, the covariances between opposing individual beams, $\rho_{u w}, \rho_{u}$, and $\rho_{w}$, can be expressed as:

$$
\begin{gathered}
\overline{v_{3}^{\prime} \dot{w}_{1}}=\overline{v_{1}^{\prime} w_{3}}=\rho_{v w} \overline{\bar{v} w^{\prime}} \\
\overline{v_{3} \dot{v}_{1}}=\overline{v_{1}^{\prime} v_{3}^{\prime}}=\rho_{v} \overline{\hat{v}^{2}} \\
\overline{w_{3} \bar{w}_{1}}=\overline{\bar{w}_{1} \dot{w}_{3}}=\rho_{w} \overline{\bar{w}^{2}}
\end{gathered}
$$

Thus, the correlation-corrected velocity variances are given by Newman et al [42] as:

$$
\begin{aligned}
& \overline{\hat{u}_{D B S-\text { corr }}^{2}}=\frac{2}{1+\rho_{u}} \overline{\hat{u}_{D B S}^{2}}-\frac{\left(1-\rho_{w}\right) \sin ^{2} \phi}{\left(1+\rho_{u}\right) \cos ^{2} \phi} \overline{w^{2}} \\
& \overline{\hat{v}_{D B S-c o r r}^{2}}=\frac{2}{1+\rho_{v}} \overline{\hat{v}_{D B S}^{2}}-\frac{\left(1-\rho_{w}\right) \sin ^{2} \phi}{\left(1+\rho_{v}\right) \cos ^{2} \phi} \overline{w^{2}}
\end{aligned}
$$

where $\hat{u}_{D B S}^{2}$ and $\hat{v}_{D B S}^{2}$ are the variances of earth coordinate velocities obtained using the DBS method from equations (3.7). Auto-correlation coefficients were determined by Newman et al [42] by using two sonics placed $11.5 \mathrm{~m}$ apart, compared to scanning diameter of lidars ranging 70 to $220 \mathrm{~m}$. The coefficients for covariances were computed separately for convective and stable conditions. Due to measurement limitations, similar auto-correlation measurements were not available here. For a more simplistic evaluation of this potential correction, the same correction factors as in Newman et al [42] were utilized. During the convective conditions $\rho_{u}, \rho_{v}$, and $\rho_{w}$ were taken to be $0.96,0.81$, and 0.66 while under stable conditions were taken to be $0.95,0.71$, and 0.69 respectively. TI and TKE were calculated by equations (3.1b) and (3.1c) respectively by using $\dot{u}_{D B S-c o r r}^{2}, \hat{v}_{D B S-c o r r}^{2}$, and $\overline{w^{2}}$. 


\subsection{Data Processing}

A number of quality control and quality assessment steps were taken to ensure the data from all sensors were reliable and cleaned of noise or poor quality measurements. All sensors measured ensemble averages either on 10 or 20-minute time intervals throughout the study period. Quality control steps for each sensor type are described below.

\subsubsection{Lidar}

All the data for a height when raw $(1 \mathrm{~Hz})$ CNR was less than $23 \mathrm{~dB}$ was removed for the beam in question. In each 10 minute burst, the CNR, radial wind speed and radial wind speed dispersion were passed through a first difference check. Data outliers (greater than three times the standard deviation) were identified and all the data at the height and time were removed. This was repeated for three iterations. After removing outliers, 10-minute bursts with less than $75 \%$ data return were omitted. In each remaining viable 10-minute burst (Tab. A.1), the variance of the radial wind speed for each beam was computed. Velocity variances were computed from the horizontal velocity components, estimated using DBS from the radial velocities measured by the WindCube v2.0, as was the mean of the horizontal wind speed and wind direction. TI and TKE were computed using the three methods (for each lidar turbulence estimation technique: the DBS method, the DBS-corrected method (equations: 3.9), and the EB-5 method (equations: 3.5a and 3.5b). When analyzing TI, times with mean winds $<1 \mathrm{~m} / \mathrm{s}$ and TKE value $>12 \mathrm{~m}^{2} / \mathrm{s}^{2}$ were removed for

quality control concerns. When comparing the mean profiles of wind speed, TI and TKE, the standard error of mean (error bar) was computed by dividing the standard deviation of the time series data by the square root of effective degrees of freedom, which was estimated as number of unique days in the data. 


\subsubsection{Sonic anemometer}

The $20-\mathrm{Hz}$ raw sonic data from both sonic anemometers was available in 20-minute long burst files. The data for each file was split and grouped into two $\sim 10$ minute bursts. In each burst, times where any of the velocity component was greater than three times the standard deviation was identified and the data removed. This was repeated for three iterations. Data with spikes in vertical velocity and standard deviations greater than $1 \mathrm{~m} / \mathrm{s}$ were removed. Bursts with less than $90 \%$ data were removed from further analysis. In each remaining burst (Tab. A.1), the variance of each velocity component, as well as the standard deviation and the mean of the horizontal wind speed were computed and used to estimate TKE and TI as described above (Section: 3.3). The two sonic anemometers were located on the east and west side of the ASIT tower respectively and the burst averaged data from the sonic anemometer aligning with the time-dependent wind direction (i.e. the direction the wind is coming from) was used to form a single timeseries of the results in order to avoid a bias due to the wake of the tower. Additionally, a small subset of bursts remained, generally oc-

curring during high wind speed events, in which the combined sonic product greatly over-estimated variances, compared to lidar or cup anemometers. This suggested an additional influence of the tower or structure on turbulence estimates observed by the sonic anemometers. To eliminate these periods, sonic-based TKE data where the difference between the sonic anemometer and the lidar TKE (using any of the three methods) was greater than three times the mean TKE was considered to be an outlier and removed.

\subsubsection{Cup anemometers and wind vane}

The $1 \mathrm{~Hz}$ raw data was grouped into 10-minute bursts. Similar to the lidar and sonic observations, raw data greater than three standard deviation away from the mean was removed, for each of the three iterations for each burst. For the remaining bursts (Tab. A.1), the mean horizontal wind speed, wind direction and standard deviation of horizontal wind speed were computed for each burst and TI was computed using 
equation 3.1a. TI was not estimated for bursts (for both sonic and cup anemometers) where mean horizontal speed was less than $1 \mathrm{~m} / \mathrm{s}$ due to the mathematical bias of the formula used [47].

\subsubsection{Atmospheric Stability}

The processed 20-minute burst averaged measurements of air temperature, pressure, relative humidity, sea surface temperature, friction velocity and buoyancy flux available for the first half of the study period (November to February) were used to directly estimate the Obukhov length scale as:

$$
L=\frac{-u_{*}^{3} \overline{\theta_{v}}}{\kappa g \overline{w^{\prime} \theta_{v}^{\prime}}}
$$

where: $u_{*}$ represents friction velocity, $\kappa$ is the Von Karman contant $(\sim 0.41), g$ is the gravitational acceleration, $\theta_{v}$ is the virtual potential temperature, and $\overline{\bar{w}^{\prime}}$ is the mean buoyancy flux. A burst was classified as stable if $L$ was between 0 and $600 \mathrm{~m}$, convective if $L$ was between 0 and $-600 \mathrm{~m}$, and neutral if the absolute value of $L$ was greater than $600 \mathrm{~m}$. During the measured period for this processed dataset, there were only a small number of bursts $(<10)$ which could be classified as neutral. Hence, neutral conditions were not included in the results. The same stability classification from the 20-minute burst was used for the resulting two 10-minute bursts.

For the second half of the study period, spanning February to June 2020, the stability was estimated more crudely via the difference between the air temperature (measured at $20 \mathrm{~m} \mathrm{amsl}$ ) and water temperature (at $4 \mathrm{~m}$ below $\mathrm{msl}$ ) due to unavailability of sufficient observations to estimate $L$ directly during this period. Conditions were inferred to be convective if the temperature difference (air temperature - water temperature) was negative and stable if the difference was positive. Testing the validity of this approximation against $L$ during the first half of the study period, using a similar approximation during that period, only $13 \%$ of the stability classification was different using the air-sea temperature difference as the indicator, compared to that possible using $L$ itself. Use of the air-sea temperature difference to categorize 
changes in TKE or TI had no appreciable change on the results presented here. Thus, while less than optimal, the air-sea temperature difference was used to categorize atmospheric stability during the second half of the study period in the results presented here. Table A.2 shows the stability classification during both periods. 


\section{Chapter 4}

\section{Results}

The results presented here are divided into four sections. In the first section, the mean prevailing conditions in the region during the deployment period are presented. In the second and third sections, the Turbulence Intensity and Turbulent Kinetic Energy estimated by the three instruments as well as the three computational techniques for the lidar are compared. The fourth section examines the time variability of turbulence observed by the lidar over the course of the study period. Throughout, the results in convective and stable atmospheric conditions are also compared.

\subsection{Mean conditions}

Over the full deployment period, the lidar (at $53 \mathrm{~m} \mathrm{amsl)}$ and the cup anemometer (26 m amsl) showed similar wind speed patterns, with a 40-hour low pass filtered time series (to remove diurnal or shorter variations) of both having numerous wind events occurring at time periods of 3-7 days. Mean winds over the study period spanned from wind speeds near zero to as high as $18 \mathrm{~m} / \mathrm{s}$ (Fig. B-3). The mean horizontal wind speed averaged over the study period was $\sim 9 \mathrm{~m} / \mathrm{s}, \sim 10 \mathrm{~m} / \mathrm{s}$ and $\sim 10.9 \mathrm{~m} / \mathrm{s}$ at $53 \mathrm{~m}, 120 \mathrm{~m}$ and $180 \mathrm{~m}$ respectively. Lidar-based wind speed at $180 \mathrm{~m}$ had $\sim 21 \%$ more gaps than at lower heights, due to reduced data return at the higher heights and from the quality control measures described in Section 3.4. The lidar's CNR value, a metric of data quality, generally decreases with height. Removing more than $30 \%$ of 
the points from a 10-minute burst because of quality control measures can void the speed for the entire burst. However, this reduction in data availability with height did not effect the mean wind speed estimate given here or the turbulence results shown below.

Wind direction measured by lidar (Fig: B-3) was similar at all heights (correlation $(R)$ of 0.98 between $53 \mathrm{~m}$ and $180 \mathrm{~m}$ ), generally veering less than $10^{\circ}$ over a 127 m vertical separation on 40-hour time scales (Fig: B-3). These results suggest that winds were predominantly unidirectional with only small veer during the study period, although there could be more variability in the wind direction for time scales less than 40-hour.

When the deployment period was divided into two periods: November to February (roughly characterized as winter conditions) and March to June (roughly characterized as spring-like conditions) (Fig: B-4), the predominant wind direction changed to be from West-northwest to Southwest (in meteorological convention). The 40hour low pass filtered wind speed (Fig. B-3) measured by lidar at 53, 107, and 167 m (Fig. B-3) were higher during spring compared to winter: $\sim 8.9 \mathrm{~m} / \mathrm{s}, \sim 9.8 \mathrm{~m} / \mathrm{s}$, $\sim 10.6 \mathrm{~m} / \mathrm{s}$ during winter and $\sim 9.2 \mathrm{~m} / \mathrm{s}, \sim 10.4 \mathrm{~m} / \mathrm{s}, \sim 11.4 \mathrm{~m} / \mathrm{s}$ during spring. The cup anemometer based mean wind speed distribution did not show any significant difference between the two seasons, but the median wind speed was slightly higher during spring $(\sim 7.8 \mathrm{~m} / \mathrm{s}$ for spring and $\sim 7.2 \mathrm{~m} / \mathrm{s}$ for winter). The distribution of TI had similar patterns in both seasons but with a slightly narrower distribution in the spring. The distribution of TKE from the sonic anemometer in Spring showed a positively skewed distribution with a median of $\sim 0.27 \mathrm{~m}^{2} / \mathrm{s}^{2}$. A previous study done in 2017 [3] at this site, showed that the turbulence characteristics, via estimates of the turbulent dissipation rate, for summer and spring varied due to the direction of wind, and whether it was approaching the ASIT from land or sea. When the data was divided based on wind direction (meteorological convention) (Fig: B-5), the median for the wind speed for wind blowing from sea was slightly higher compared to that blowing from land $(\sim 7.46 \mathrm{~m} / \mathrm{s}$ for land and $\sim 7.64 \mathrm{~m} / \mathrm{s}$ for sea). The distribution of sonic TKE showed a broader distribution for the wind blowing from land compared 
to the wind blowing from sea with dramatically different median TKE $\left(0.51 \mathrm{~m}^{2} / \mathrm{s}^{2}\right.$ for land and $0.2 \mathrm{~m}^{2} / \mathrm{s}^{2}$ for sea).

Despite estimating the Obukhov length for the first half but using a rudimentary method to estimate stability in the second half (discussed in Sec: 3.4), the number of convective events and stable events appeared to be equally distributed throughout the study period (Tab. A.2). Note that the period between February 12 and 28 had no meteorological data to determine the stability, and is excluded from comparisons based on stability.

In general, the mean wind speed profile from the lidar (Fig. B-6), illustrated that the wind speed almost linearly increased with height. When the wind speed was divided into three subsets based on percentiles (Fig. B-6), only the highest percentile showed an increasing trend while the vertical structure is predominantly uniform with height. When the mean profile was compared for convective and stable conditions (Fig. B-6), the mean wind speed profile during stable conditions showed significant mean shear, defined here as a speed difference over $147 \mathrm{~m}$ vertically, $\sim 3.2 \mathrm{~m} / \mathrm{s}$ between $53 \mathrm{~m}$ and $200 \mathrm{~m}$ amsl and a mean shear of only $\sim 1 \mathrm{~m} / \mathrm{s}$ during convective conditions. The TI measured by cup anemometer (Fig. B-7) was a positively skewed distribution with TI generally being less than 0.2. The TI- wind speed comparison (Fig. B-7) showed that larger TI $(>0.2)$ occurred mostly during low wind speed conditions $(<$ $0.5 \mathrm{~m} / \mathrm{s}$ ). The large TKE values could be either be due to larger fluctuations at lower wind speeds or just due to normalization with low mean wind speed. The shape of the distribution for TI cup vs. wind speed $(\mathrm{y}=1 / \mathrm{x})$ suggests that the latter could be the reason. Hence, when utilizing TI for turbulence characterization at low wind speeds, this bias should be taken into account.

\subsection{Turbulence Intensity (TI) validation}

A comparison of the two TI conventions (industry and meteorology) between the cup anemometer and both the sonic and the bottom bin (53 m amsl) of the lidar (Fig. B8) revealed that use of either convention achieves similar results. The two conventions 
changed the TI only slightly (slope difference less than 0.02 and correlation coefficient difference less than 0.05) for both lidar DBS method and sonic anemometer. Although the industry definition showed a slightly higher $R$, the meteorological definition was used for all the analysis here onwards by dropping the met suffix due to its theoretical robustness.

A TI comparison between the three lidar methods (Fig. B-9) revealed that TI DBS and TI DBS-corrected generally had similar magnitude as TI cup (linear regression slopes of $1 \pm 0.03)$, although the lidar sampling volume was almost $26 \mathrm{~m}$ higher than the cup sample volume. The EB-5 method showed a generally lower TI compared to TI cup with a slope of 0.72 . Note that the mean wind speed used in the TI calculation was equal for all the three methods (Equation. 3.1b) and that the mean wind speed decreased with height (Fig. B-6). Thus, if the turbulence was constant with height, the TI would decrease with height, which would have resulted in lower TI at lidar sampling heights compared to the cup sampling height of $26 \mathrm{~m}$ amsl.

Sonic anemometer had the lowest slope in the TI comparison (Fig. B-9) although cup anemometer was only $6 \mathrm{~m}$ higher than sonic anemometer. However, the bin averaged TI showed that there was good agreement between the two at $\mathrm{TI}<0.2$. A majority of the cup TI lie in this low TI range (Fig. B-7) while the high TI values mostly occurred at low wind speed (Fig. B-7). Thus, this bad fit was driven by high TI values which occurred at low wind speeds. This is further discussed in Ch.5.

Note that the data available for lidar and cup anemometer is almost twice the amount of data available for the sonic anemometer deployment, which is available only for spring. For statistical robustness, the TI comparison was also done just for the spring period (results not shown here). The TI-fit slopes decreased slightly by 0.06 and 0.02 for both DBS methods and EB-5 method respectively, while correlation decreased by 0.02 for the three methods. Hence the data availability differences between sonic and lidar does not change the results significantly. The comparisons of the lidar methods and sonic anemometer (Fig. B-10) during stable and convective conditions, did not change the interpretation from the full period. The slope for all the methods was slightly higher in stable conditions compared to convective conditions, while 
also having higher correlation during stable conditions. The DBS methods generally showed higher correlations with the cup anemometer, having linear regression slopes less than 1 in convective and more than 1 in stable.

\subsection{Turbulent Kinetic Energy (TKE) validation}

The absolute value of the lidar TKE estimated by the DBS methods were similar to the TKE estimated by the sonic anemometer data-set, while the EB-5 based TKE estimate generally had a lower TKE (Fig: B-11) than observed by the sonic anemometer. The DBS method and the DBS corrected method had slopes slightly higher and lower than 1 respectively while having similar $R$. The DBS correction appeared to reduce the slope of a linear regression by around $9 \%$ and decreased the $R$ negligibly. The DBS method had a small positive offset from the slope 1 line while the correction further reduced the offset (offset value not shown). The EB-5 method had the lowest $R$ and the spread in data increased with TKE. The slope is lower by $24 \%$ compared to DBS method while $R$ decreased by $8 \%$ compared to the DBS method. This suggested that DBS methods estimated TKE at $53 \mathrm{~m}$ amsl to be equal to TKE at $20 \mathrm{~m}$ amsl while the EB-5 method estimated a TKE at $53 \mathrm{~m}$ amsl that was lower than at $20 \mathrm{~m}$ amsl. Given equal levels of turbulence, lidars sample a scanning area which averages the small scale turbulent features and hence one would expect the lidar to estimate lower turbulence levels than that possible via a sonic anemometer, which is effectively a point measurement. This is further discussed in Ch.5.

A linear regression fit minimizes the error of the dependent variable from the linear fit. This inevitably gives more weight in reducing the errors at higher TKE values than at lower TKE values where the majority of the points were located, as noted by Bonin et al [47] and Bodini et al [50]. Since the TKE values can range two orders of magnitude, this could lead to a biased regression and lower $R$ values. Therefore, the slope and $R$ value of linear regressions fit are potentially not the best

metrics to compare the lidar TKE methods against an independent standard (here the sonic TKE). The effect of the high range of TKE, spanning orders of magnitude, 
can be partly accounted for by taking a log transform (base 10) of the TKE values and comparing the linear fit of the log values, which would normalize all orders of magnitude more equally.

The log-transformed TKE (Fig. B-12) showed less spread and improved fit to the linear regression for all lidar TKE methods compared to the non-transformed lidar TKE (Fig. B-11). The DBS method had a slope closer to 1 than the DBS corrected method although $R$ is the same. The EB-5 method had a slope near 1 with an improved $R$, but slightly less than the DBS methods. Log transformed TKE for DBS method had a decreased slope by $12 \%$ compared to the non-log transformed fit, while $R$ increased slightly by $0.6 \%$. Log transforming the EB-5 TKE values improved the slope by $25 \%$ and increased $R$ by $6 \%$. This significant improvement in performance for EB-5 for log-TKE values and under-performance by DBS for log-TKE values suggests that the change in agreement for both was related to the relative weighting of TKE events on the fit, compared with low TKE events (Fig. B-11). When TKE values were given more equal importance for all orders of magnitudes, the EB-5 method gave a consistently lower values at all times, a slope of one, and improved correlation to the sonic TKE dataset.

When the TKE methods are compared for the two stability conditions (Fig. B13), the DBS methods performance did not show remarkable differences between the two stability conditions. In contrast, the EB-5 method resulted in a decrease in slope by $11 \%$ in stable conditions compared to convective conditions. This decrease was in line with that expected as stable conditions have less turbulence, for a given wind strength, when compared to convective conditions. However, the nuanced difference in performance for varying stability regimes suggests that the EB-5 method might be better at distinguishing between the two regimes than DBS-based methods which do not appear to vary with stability. This difference might be the result of increased noise in the DBS-based estimates. The log-TKE regression results (Fig. B-14) appeared to further support this argument. Under both the stability conditions, DBS methods at lower TKE values are elevated in comparison to the sonic TKE. At higher TKE values they are reduced relative to the sonic's TKE. Again, with no difference in 
these trends with stability, the DBS-based methods appeared to be insensitive to the atmospheric stability conditions. In contrast, the EB-5 method had a log-linear slope of $\sim 1$ and a constant offset in TKE (a reduction) relative to the sonic's log-TKE. The negative bias seen under stable conditions here could be due to the smaller turbulent length scales that are more likely to be present during stable conditions being partially underestimated by the sample volume of the lidar. These differences will be discussed further in the Ch.5.

\subsection{Mean Turbulence Conditions}

An examination of time series of TI for both the DBS and EB-5 methods for the full study period, filtered using a filter with a 40-hour half power response, illustrated the vertical and temporal variability in TI. Comparing TI at heights of $53 \mathrm{~m}$ and $180 \mathrm{~m}$ (Fig. B-15) showed that the TI at $53 \mathrm{~m}$ is highly correlated $(R>0.92)$ with TI at $180 \mathrm{~m}$. Differences in the magnitude of TI at these heights, separated by $127 \mathrm{~m}$, were seen only during strong turbulent events (seen in Fig. B-15 at peaks) where the TI at $180 \mathrm{~m}$ was slightly higher than at $53 \mathrm{~m}$. The most notable difference between TI timeseries comparisons for the DBS and EB-5 methods was that the EB-5 method results in estimates that are slightly lower in TI values. However, this difference was not large, and no significant differences existed in the time variability of TI for either method. For both methods, the timescale of strong TI events seems to be around a few days to a week.

The vertical structure of TI, averaged over the full study period, as well as the convective and stable conditions separately, was mostly uniform with height over the range of the lidar (Fig. B-16). For all conditional averages, TI increased with height as much as $10 \%$ between the $53 \mathrm{~m}$ and $80 \mathrm{~m}$ before decreasing with height thereafter up to $200 \mathrm{~m}$. This pattern was similar for both the stability conditions as well as for DBS and EB-5 based results. EB-5 based TI estimates were lower than DBS for all conditions; and TI estimates during stable conditions were lower than convective for both methods. In all cases, the change in the height averaged mean TI 
between stability conditions or TI methodologies was larger than the change in the vertical structure itself. The decreasing TI with height above $80 \mathrm{~m}$ (Fig. B-16) might illustrate one of the limitations for using TI normalized by wind speed for turbulence studies spanning a range of heights above the boundary. A large part of this measured decrease with height occurred because the wind speed, which normalizes the turbulent fluctuations in TI, increased with height (Fig: B-6). Note also that the data from $120 \mathrm{~m}$ upwards was always lower vertical resolution $(20 \mathrm{~m})$, than that between 80 and $120 \mathrm{~m}$, as the instrument was originally commissioned to have finer scale resolution around the likely hub height of a wind turbine.

TKE time series for DBS and EB-5 methods at $53 \mathrm{~m}$ and $180 \mathrm{~m}$ were also highly correlated ( $R=0.98$; Fig. B-17) with highly similar magnitudes and fluctuations, demonstrating that TKE was generally nearly constant with height. Differences in the vertical structure of TKE were most significant during strong TKE events (seen as peaks in Fig. B-11). Differences in TKE between the two heights during strong TKE events were more prominent than the differences seen in TI at these heights (Fig. B15). For example: the strong event in the beginning of March (Fig. B-17) had more than twice the TKE at $180 \mathrm{~m}$ compared to $53 \mathrm{~m}$. Yet this sizable difference was not visible in the TI time series (Fig: B-15). Similar to TI, the TKE values were lower using the EB-5 method compared to the DBS method, particularly at the higher, or peak, events. The vertical structure of TKE using DBS, averaged over all times, increased non-linearly to $140 \mathrm{~m}$ height, before then decreasing with height (Fig. B-18) - The vertical structure during both convective and stable conditions was similar to the full period mean, but had higher TKE values during the convective conditions. The EB-5 method results also had a vertical structure with a local maximum at 140 m, but again, with lower TKE values compare to the DBS method.

As discussed in Sec.4.1, convective and stable events were almost equally distributed during the study period. As strong TKE events occurred sporadically with TKE values an order of magnitude larger than the rest of the period, averaging in time (over convective or stable conditions) had the potential effect of eliminating any structure in the mean profile. Dividing the stable and convective TKE results into 
four separate bins based on the height-averaged TKE revealed the differences in the vertical structure of TKE during weak, moderate, and strong TKE events (Fig. B19). For the lowest two TKE bins, averaging profiles for height-averaged TKEs of 0-0.4 $\mathrm{m}^{2} / \mathrm{s}^{2}$ and $0.4-1.0 \mathrm{~m}^{2} / \mathrm{s}^{2}$, the bin averaged TKE vertical structure appeared to be quasi-uniform in height. For the bin with moderate height-averaged TKE values (1.0 to $\left.2.0 \mathrm{~m}^{2} / \mathrm{s}^{2}\right)$, the bin averaged TKE profile showed a modest increase $(\sim 0.25$ $\mathrm{m}^{2} / \mathrm{s}^{2}$ from $53 \mathrm{~m}$ to $200 \mathrm{~m}$ ) in TKE with height. The bin with largest TKE values (> $\left.2 \mathrm{~m}^{2} / \mathrm{s}^{2}\right)$, increased in TKE with height up to $160 \mathrm{~m}\left(\sim 0.8 \mathrm{~m}^{2} / \mathrm{s}^{2}\right)$ before decreasing slightly to $200 \mathrm{~m}$. This largest bin also had the highest difference between stable and convective conditions $\left(\sim 0.25 \mathrm{~m}^{2} / \mathrm{s}^{2}\right)$ which was nearly constant with height.

The lowest TKE bin $\left(0-0.4 \mathrm{~m}^{2} / \mathrm{s}^{2}\right)$ of the conditionally-averaged TKE vertical structure, small in comparison to the larger, high-TKE event bin averages, had a notable vertical structure. While the vertical structure appeared to be uniform, with error bars for this bin too small to be seen, in Fig. B-19, the vertical structure of the smallest bin had a statistically significant non-linear structure with a minimum around $120 \mathrm{~m}$ (Fig. B-20). As described in Sec. 3.4.1, the standard error of mean for conditional averages were estimated by dividing the standard deviation by the square root of the independent degrees of freedom, taken to be number of unique days in each bin. The magnitude of the vertical variations was up to $20 \%$ of the mean value for all conditional averages shown, and was more prominent during the convective conditions for both DBS and EB-5 methods. Importantly, the smallest bin shown here, sorted by the height-averaged TKE, encompassed $30 \%$ of all data during convective conditions, and $58 \%$ of all data during stable conditions. Thus, during the bulk of the observational periods for both types of stability conditions, TKE was fairly small in magnitude, but had this significant non-linear vertical structure with a local minimum at 120-140 $\mathrm{m}$ amsl. 


\section{Chapter 5}

\section{Discussions and Conclusion}

As the energy sector pivots towards renewable energy, the wind energy industry is expanding around the world. Offshore wind energy offers a unique advantage of higher power generation due to higher wind speeds as well as the ability to install larger turbines. The few studies that have been done in offshore environments show that the turbulent properties of the wind in offshore sites-which determine the power generation, wake properties, life cycle costs, and structural strength of the wind turbine-are notably different from more well-researched onshore sites. Difficulty in the deployment of meteorological instrumentation at turbine heights in offshore environments has given way to a dependence on remote sensing instrumentation for both the general characterization of the wind resource as well as estimates of the turbulent properties of the boundary layer. In addition to improved vertical coverage, remote sensing instruments are cost effective and easily deployed, relative to met towers. In the past decade, several land-based studies and a smaller number of offshore-based studies have shown lidars to be useful in measuring turbulent properties of the atmospheric boundary layer. The fundamental difference in the measuring principles between remote sensing devices and more traditionally used in-situ instruments like cup and sonic anemometers has resulted in a number of efforts to compare the estimates of the wind resource measured by point sampling and profiling instruments. Addition-

ally, several different measurement techniques to estimate turbulence with the remote sensing devices have been examined. This thesis is among the few that have done so 
in the marine environment, in which atmospheric properties can be distinctly different than more familiar onshore wind conditions.

This thesis examines the leading techniques to characterize the mean and turbulent conditions in the marine atmospheric boundary layer, which is of critical importance to the rapidly growing offshore wind energy industry. The data used was obtained from an offshore meteorological tower located south of Martha's Vineyard, Massachusetts which is $10 \mathrm{NM}$ from multiple wind energy lease areas. During the study period, spanning November 2019 to June 2020, a mean wind speed of $\sim 10 \mathrm{~m} / \mathrm{s}$ was observed at the heights of typical wind turbines. The major difference seen in seasonality was the change in wind direction from winds out of the West-Northwest in winter to winds from the Southeast in spring and early summer, with slightly higher mean wind speeds in the spring. The study period was predominantly characterized by low turbulence conditions with vertical variations $20 \%$ of the height-averaged mean value. Strong turbulent events had strong vertical structure. The range of TKE values observed was found to be much greater than the difference between measurement methods and instruments themselves.

In this section, the impact of the assumptions made in each of the lidar-based turbulence estimation methods is discussed, followed by a discussion of TKE and TI comparisons, the vertical structure of turbulence, and the potential uses of TKE vs. TI as a metric for turbulence in this type of dataset.

One of the most convenient and economically feasible remote sensing instruments, vertically profiling lidar systems such as the WindCube v2.0 utilized here, estimate wind speed and direction by measuring the radial velocity along the laser beam for multiple directions and ranges. To estimate turbulence, two methods are commonly used, based on either the raw along-beam radial velocity variances themselves, or estimates of the earth coordinate velocity variances after transforming the alongbeam velocities into Cartesian coordinates. The latter method assumes homogeneous flow as well as turbulent velocities over the measured area, while the former assumes that only the statistics of turbulence are homogeneous. This difference can play an important role in complex terrains [6] where the mean flow is not likely homogeneous. 
The two methods used here, DBS and EB-5, use the earth coordinate velocity variance and along-beam velocity variance respectively.

There are multiple factors affecting TKE estimates by lidar methods, and some can have competing effects on the results. For most vertically profiling lidar systems, the scanning circle increases with height, causing both increased spatial averaging or smoothing of the turbulent length scales present, but also variance contamination due to the in-homogeneity of the flow field [42]. Area-averaging assumes that both the turbulence and the background mean flow are uniform in the circular area. This homogeneous flow assumption is partly circumvented in the EB-5 method, making it more likely to be consistent in both convective and stable conditions than the DBS methods (Fig. B-14). When the scanning circle increases with height, these spatial homogeneity assumptions can become less robust and potentially break down. However, during convective conditions, the TKE and consequently the eddy sizes may increase with height, which to some extent, may scale with the increase of the sample volume averaging area that assumes spatial homogeneity. Variance contamination [51] is a consequence of assuming that the instantaneous velocity is constant across the scanning circle area during the scanning period and transforming the individual radial velocities to earth coordinate velocities. In reality, there is some correlation between consecutive measurements which can lead to over-estimation of TKE. In theory, this can be corrected in the DBS method with a correction term, which previous studies have shown leads to a significant reduction in the TKE estimates [42] relative to the uncorrected estimates. But, it should be noted that the correction term itself is challenging to fully assess with direct observations. Thus, it is unlikely that most observational efforts would be able to utilize a more locally tuned correction method than that used here.

The results shown in this thesis indicate there is little difference in the meteorological convention and the industry convention for estimates of TI (Fig. B-8). Although there is ambiguity over the definition of the meteorological convention used in turbulence studies with lidars, the differences in instruments and methods can arise due to different measuring principles as well. When validating the TI estimated by 
lidar against cup anemometer (Fig. B-9), a difference in the measurement height of $27 \mathrm{~m}(53 \mathrm{~m}$ vs. $26 \mathrm{~m})$ is significant by itself, and can lead to different estimates of TI. The turbulence profiles generally had weak vertical structure (Fig. B-18), except during high turbulent conditions where turbulence increased with height (Fig. B-19). In addition to turbulence, as the wind speed generally increased with height (Fig. B-6), normalizing turbulence (standard deviation of wind speed) by mean wind speed resulted in decreasing TI with height. This is likely to be true for majority of the time, except during high turbulent conditions, where turbulence was observed to increase with height as much as wind speeds. Hence, the TI comparison between the cup anemometer and lidar (Fig. B-9) suggest that the EB-5 method might provide a more realistic estimate of TI compared to DBS, despite DBS results having slightly higher correlations to the cup-based results. However, there are more factors to consider, including spatial averaging in convective vs. stable conditions and variance contamination in DBS vs EB-5 methods, which could change this interpretation. Due to unavailability of the in-situ measurements at any of the lidar measuring heights in this study, we cannot conclude which of the method is more accurate overall. However, the mean vertical profile of both the methods were similar (Fig. B-16) and show almost vertically uniform mean TI profiles (Fig. B-16), despite a small bias between the two methods.

TI results from the sonic anemometer data were generally lower than the TI observed by the cup anemometer (Fig. B-9), by a margin that was more than expected given the small, 6-m, difference in vertical spacing of the instruments. This could be due to either lower standard deviation measured by the sonic anemometer or the cup anemometer under-estimating the mean wind speed due to slower response time, resulting in a higher TI than the sonic anemometer. The latter reason is more likely than the former. All TI measured by cup anemometer greater than 0.2 , where most of the disagreement occurred (Fig. B-9), were during low wind speed events ( less than $5 \mathrm{~m} / \mathrm{s}-$ Fig: B-7). At low speeds, cup anemometers might have reduced accuracy due to both the intermittent nature of wind forcing at low speeds and the cup's sampling method. Thus, it is more likely that the low wind speed conditions are causing the 
cup anemometers to over-estimate the TI by under-estimating the mean wind speeds.

The TKE comparisons shown here reveal that a wide range of TKE magnitudes are present in the marine atmospheric boundary layer (Fig. B-11). The DBS methodbased TKE estimates, which at first appear more similar to sonic anemometer-based values in linear comparisons, show more interesting biases against the sonic-based TKE values when compared as log transformed TKE. Minimizing the weight of larger magnitude TKE values on the comparison via the log transform, the EB-5 was shown to have a consistent 1:1 relationship to the sonic anemometer results (Fig. B-12). Based on the linear regression, in log space, the EB-5 method's TKE has a constant bias against the sonic data throughout the TKE range. If both measurements were to be made at the same height, this constant bias is consistent with a potential underprediction of the true TKE due to the larger sample volume averaging used by the lidar. The larger bias seen in stable conditions for the log transformed comparisons of EB-5 TKE against the sonic TKE (Fig. B-14) is also consistent with a potential reduction in the size or length-scale of turbulence present, which the lidars would underestimate a greater fraction of due to averaging in the sampling volume.

The DBS correction method attempted here used approximate corrections from previous studies [42], due to the lack of direct observations from multiple point sensors at height, and generally reduced the estimated TI and TKE values slightly. However, the correction did not change any conclusions drawn by comparing the DBS to either EB-5 method or cup and sonic anemometers. Hence, its effect on the results were minimal. This could be due to a potential mismatch of the correction factors between this offshore site and the onshore site used by Newman et al [42]. Since deploying the instruments to measure these correction factors explicitly is not common for economical reasons, we did not further investigate this correction technique in detail.

Examining the mean vertical structure of TKE (Fig. B-18) as well as TI (Fig. B-16), suggests that atmospheric boundary layer turbulence over the ASIT was fairly uniform with height, up to $200 \mathrm{~m}$ amsl. However, TI decreased beyond $140 \mathrm{~m}$ while TKE did not decrease significantly. This leads us to question which parameter more logically represents the profile of the amount of turbulence in the atmospheric bound- 
ary layer.

TKE is defined as a weighted sum of fluctuations in all three directions, while TI only measures fluctuations of wind speed in the horizontal plane. This difference means that cup anemometers do not take vertical fluctuations into account, which are particularly important during convective conditions. This illustrates one potential limitation of using TI to gain an understanding of the origin of turbulent fluctuations and their impact on turbines. The TI distribution (Fig: B-7) showed that high TI values are present only at low wind speed events. As discussed above, this results from normalizing by the mean wind speed. When comparing the time series of TKE and TI (Fig. B-17 and Fig. B-15) high TKE events did not overlap with high TI events. The high mean wind speeds associated with high TKE events act to minimize the TI magnitudes when they are normalized by mean wind speed. At low wind speeds $(<5 \mathrm{~m} / \mathrm{s})$, the high TI values observed (Fig.B-7) are not commensurate with strong turbulence, but are an artifact of just weak mean flows. Throughout the data set, these times of high TI are associated with low TKE. This contrasting characteristics of TI and TKE make them distinctively different measures of turbulence.

The study period was characterized with predominantly (approximately half of the observations) vertically uniform, low turbulence conditions. However, numerousalmost weekly-events with an order of magnitude increase in turbulence levels also occurred (Fig. B-17). This pattern was also observed in previous studies [52]. These strong turbulent events (Fig. B-19) showed a non-linearly increasing TKE unlike the profiles during the rest of the period. This does not seem to be because of the stability conditions associated with strong TKE events (Fig. B-18). but rather is likely the result of increases in turbulent length scales with distance away from the boundary. Lidars can measure larger length scales more accurately which, all else being equal, might results in an increasing TKE with height. For the same reason, lidars cannot measure small changes in turbulent scales, and hence showed more uniform TKE during low TKE events (Fig. B-19).

The structure of the mean TKE profile with the lowest TKE values $\left(<0.4 \mathrm{~m}^{2} / \mathrm{s}^{2}\right)$ showed a weak non-linear structure (Fig. B-20) with a minimum between 120 and 
$140 \mathrm{~m}$ (hub-height of a typical wind turbine). This structure was more prominent during convective periods. Minimum TKE at $140 \mathrm{~m}$ was $\sim 8 \%$ less than the heightaveraged TKE while the TKE at $53 \mathrm{~m}$ was $\sim 12 \%$ more than the average during convective periods for DBS. This difference from the average decreases by using the EB-5 method to $\sim 7 \%$ at $140 \mathrm{~m}$ and $\sim 10 \%$ at $53 \mathrm{~m}$. When the two methods were compared above hub heights, TKE remained almost constant with height above 140 m for EB-5 but the TKE increased with height for DBS, during both the stability conditions. The standard error was computed as described in Sec.3.4.1, by dividing the standard deviation of the time series data by the square root of effective degrees of freedom, which was taken as number of unique days in the data. This conservative estimate of effective degrees of freedom resulted in relatively large standard errors which inhibited any objective inference on the dynamics observed here. However, it is noted that this very small trend was present for more than $58 \%$ of the time during stable conditions and $\sim 30 \%$ of the time during convective conditions. Although the magnitude of TKE was small, stable conditions were usually characterized by high wind speeds and wind shear (Fig. B-6). Hence this non-linear persistent structure might be of a potential importance to the wind energy industry.

In conclusion, while there are many methods to estimate turbulence characteristics using lidars, the results show that all the methods compared here are qualitatively similar. The small consistent biases between the methods are a result of different assumptions inherent in each method. Moreover, the temporal variations in the TKE itself are much higher than that due to different methodologies or instruments. Thus, it is likely that atmospheric boundary layer turbulence can estimated using lidars by either of the two methods (DBS and EB-5) compared here. In addition to being economically feasible and easy to deploy, lidars have the potential to give an insight into the vertical structure of the turbulence and mean wind speed which is essential for structural and operational design of a wind farm. 
Appendix A

\section{Tables}


Table A.1: Percentage of good data for sonic anemometer, cup anemometer and Windcube v2.0 lidar at heights shown above mean seal level.

\begin{tabular}{||l|l||}
\hline Instrument and height & Percentage of good data \\
\hline Sonic $20 \mathrm{~m}$ & 84.2 \\
\hline Cup $26 \mathrm{~m}$ & 93.2 \\
\hline Lidar $53 \mathrm{~m}$ & 80.2 \\
\hline Lidar $60 \mathrm{~m}$ & 79.6 \\
\hline Lidar $80 \mathrm{~m}$ & 81.2 \\
\hline Lidar $90 \mathrm{~m}$ & 82.3 \\
\hline Lidar $100 \mathrm{~m}$ & 81.2 \\
\hline Lidar $110 \mathrm{~m}$ & 79.6 \\
\hline Lidar $120 \mathrm{~m}$ & 78.6 \\
\hline Lidar $140 \mathrm{~m}$ & 72.9 \\
\hline Lidar $160 \mathrm{~m}$ & 66.7 \\
\hline Lidar $180 \mathrm{~m}$ & 59.3 \\
\hline Lidar $200 \mathrm{~m}$ & 50.6 \\
\hline
\end{tabular}


Table A.2: Classification of stability- percentage of stable and convective conditions during the study period

\begin{tabular}{||l|l|l||}
\hline Period & Convective percent & Stable percent \\
\hline Full deployment (11-01-19 to 06-19-20) & 50.8 & 49.2 \\
\hline Winter (11-01-19 to 03-01-20) & 51.0 & 49.0 \\
\hline Spring (03-01-20 to 06-19-20) & 53.0 & 47.0 \\
\hline November & 67.5 & 32.5 \\
\hline December & 54.7 & 45.3 \\
\hline January & 63.3 & 36.7 \\
\hline February & 60.2 & 39.8 \\
\hline March & 51.2 & 48.8 \\
\hline April & 60.7 & 39.3 \\
\hline May and June & 67.9 & 32.1 \\
\hline Day & 56.2 & 43.8 \\
\hline Night & 54.5 & 45.5 \\
\hline
\end{tabular}


Appendix B

Figures 


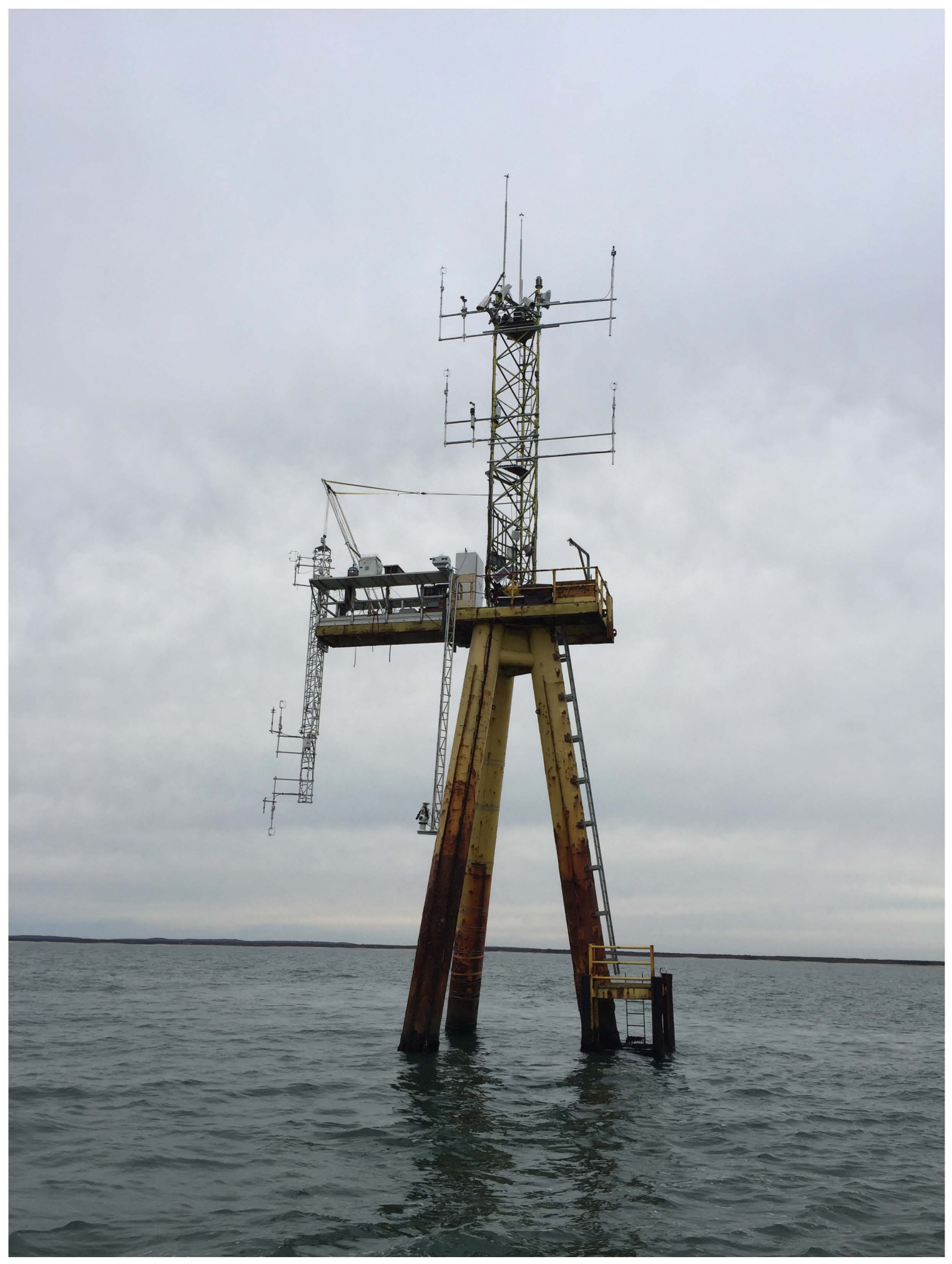

Figure B-1: ASIT met-tower. Windcube v2.0 (white box) is mounted on a platform $13 \mathrm{~m}$ above mean sea level. 


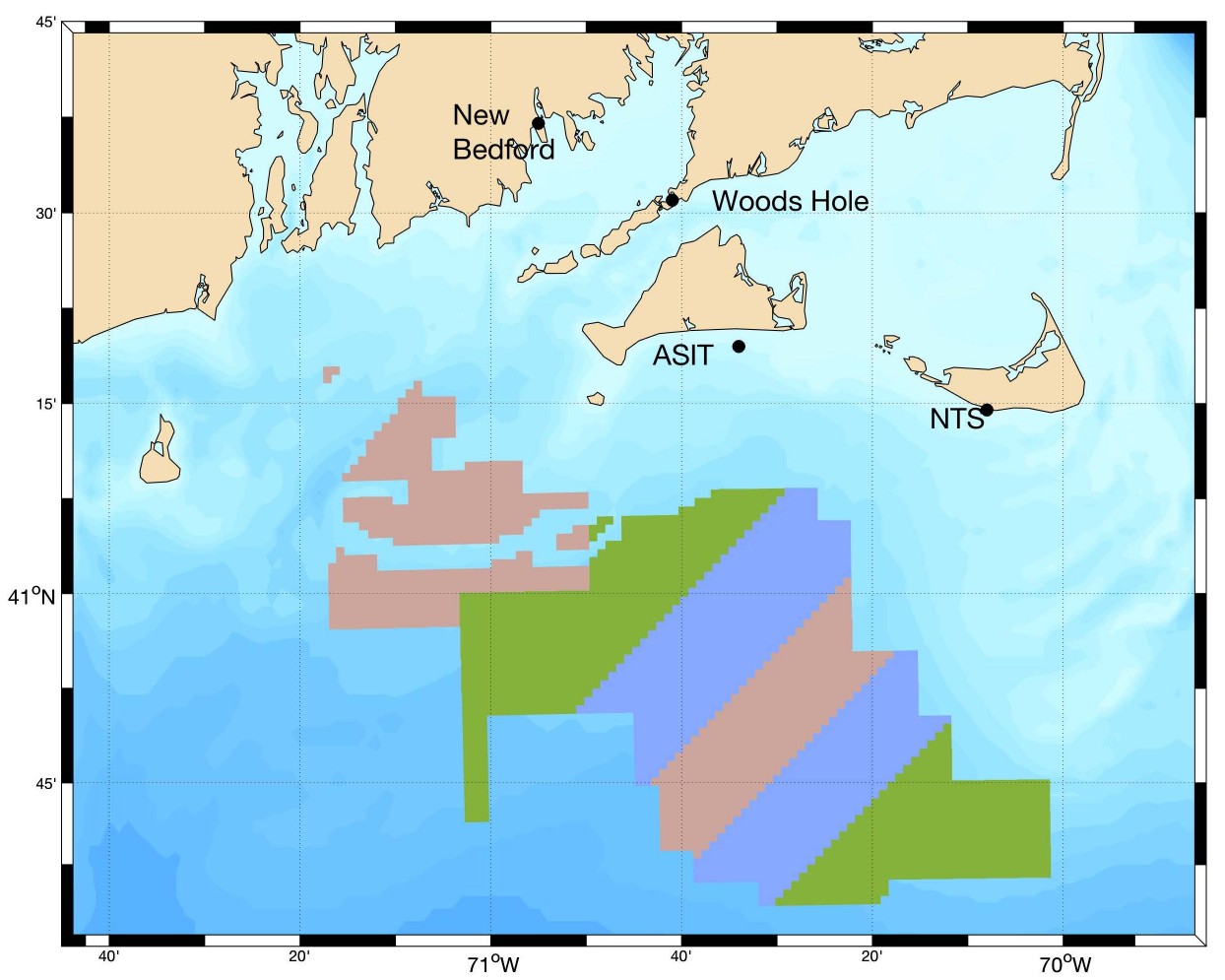

Figure B-2: Location of ASIT. Shaded area are lease areas for wind energy development 

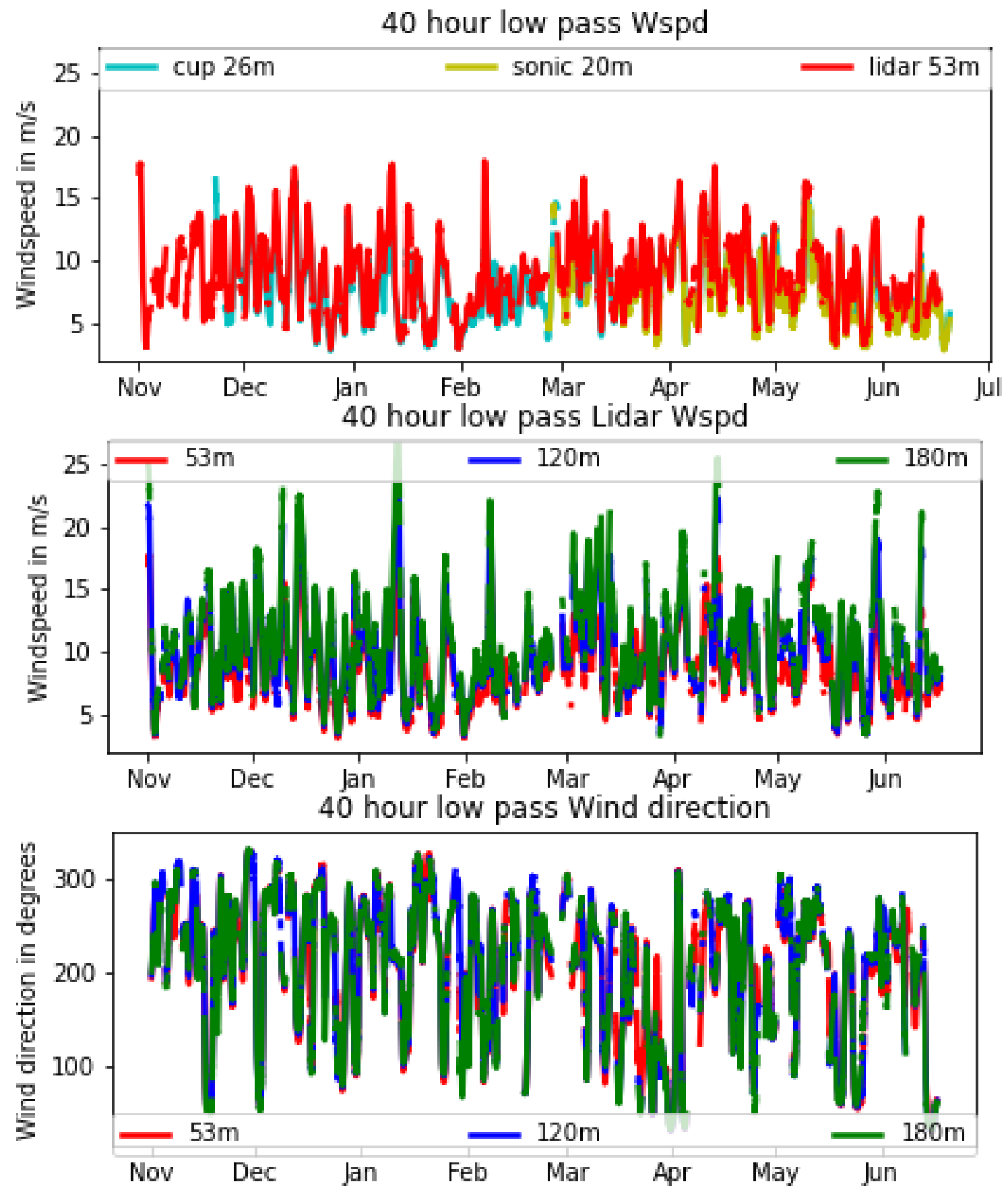

Figure B-3: Top: 40-hour low pass mean wind speed for cup, sonic anemometers and Windcube v2. Middle: 40-hour low pass mean wind speed observed by Windcube v2.0 at 53, 120 and $180 \mathrm{~m}$ Bottom: 40-hour low pass mean wind direction observed by Windcube v2.0 at 53, 120 and $180 \mathrm{~m}$. Wind direction is the direction from which the wind is coming from (meteorological convention), measured clockwise from North in degrees. 40-hour low pass filter is used to remove the diurnal effects. Sonic data is not available for the winter. 

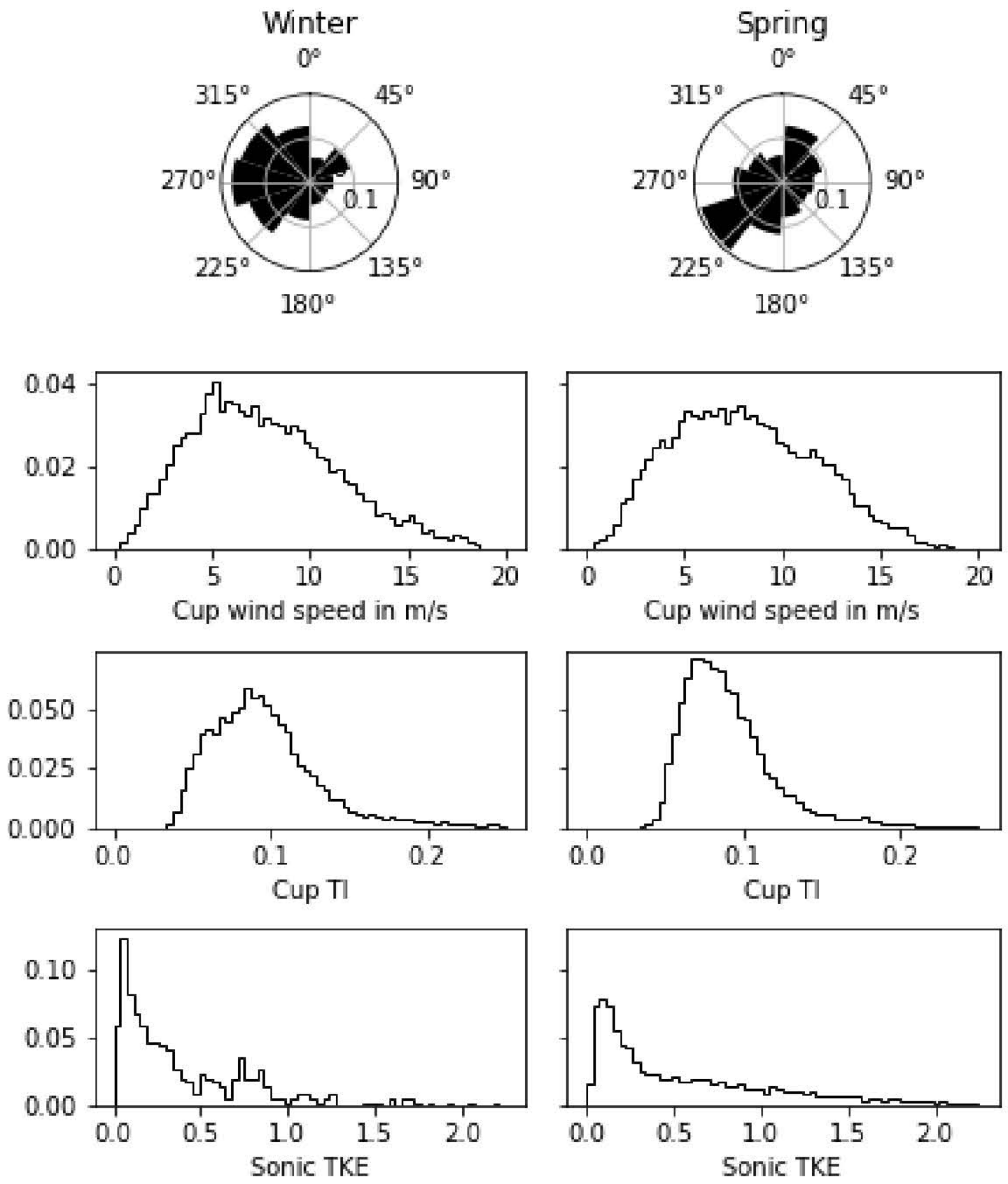

Figure B-4: Top to bottom: Distribution of wind direction, speed, and TI measured by cup anemometer and TKE estimated from sonic anemometer for Winter and Spring (before 01 March and afterwards). $\mathrm{y}$-axis is the normalized frequency and wind direction is in meteorological convention as in (Fig: B-3). Sonic data is available for only 3 days in the winter and hence the distribution is not an accurate representation for full winter period. 

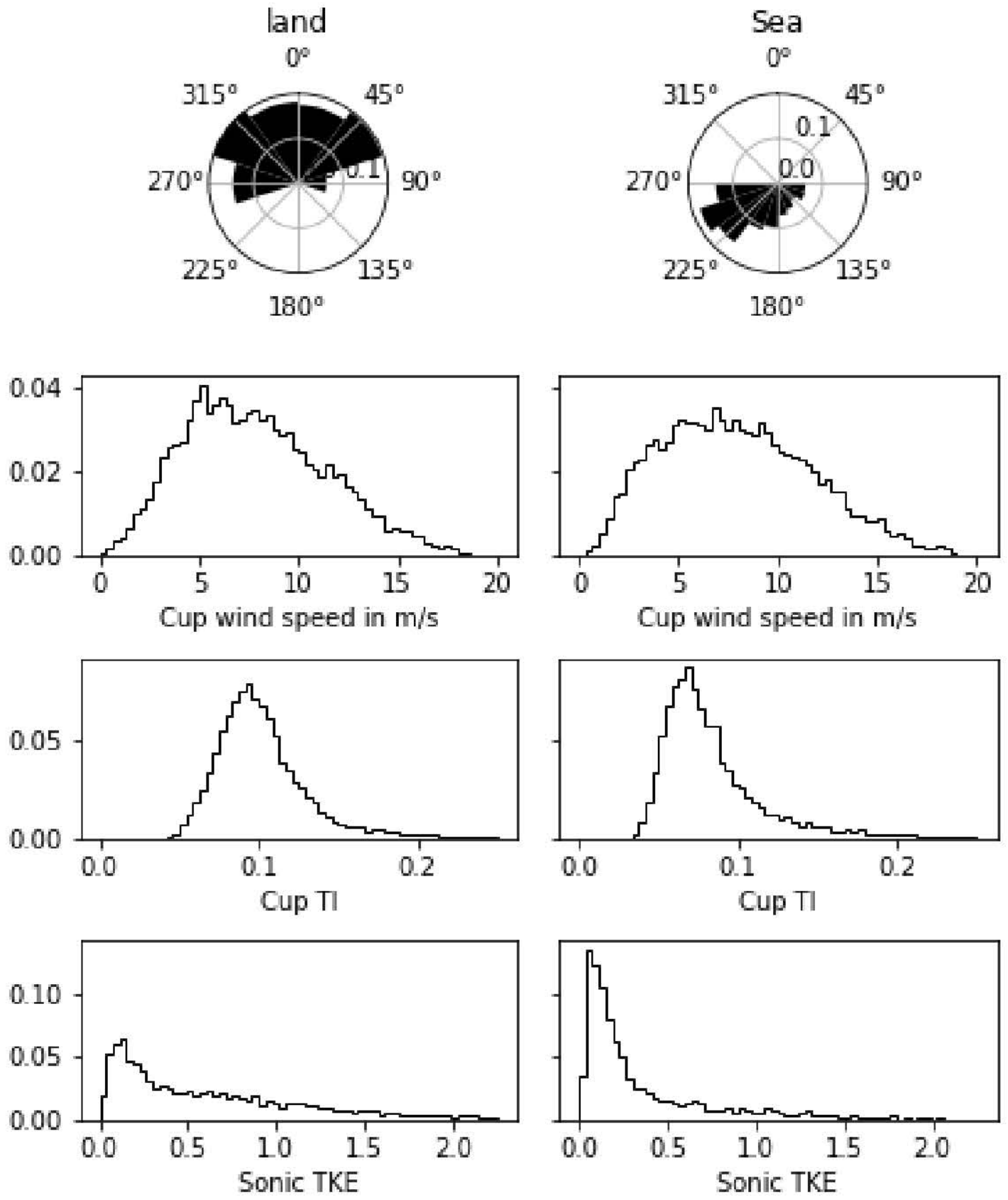

Figure B-5: Top to bottom: Distribution of wind direction, speed, and TI measured by cup anemometer and TKE estimated from sonic anemometer during times for wind coming from land (clockwise 270 to $90^{\circ}$ ) vs sea (clockwise 90 to $270^{\circ}$ ). y-axis is the normalized frequency and wind direction is in meteorological convention as in (Fig: B-3). Sonic data is available only during spring. 

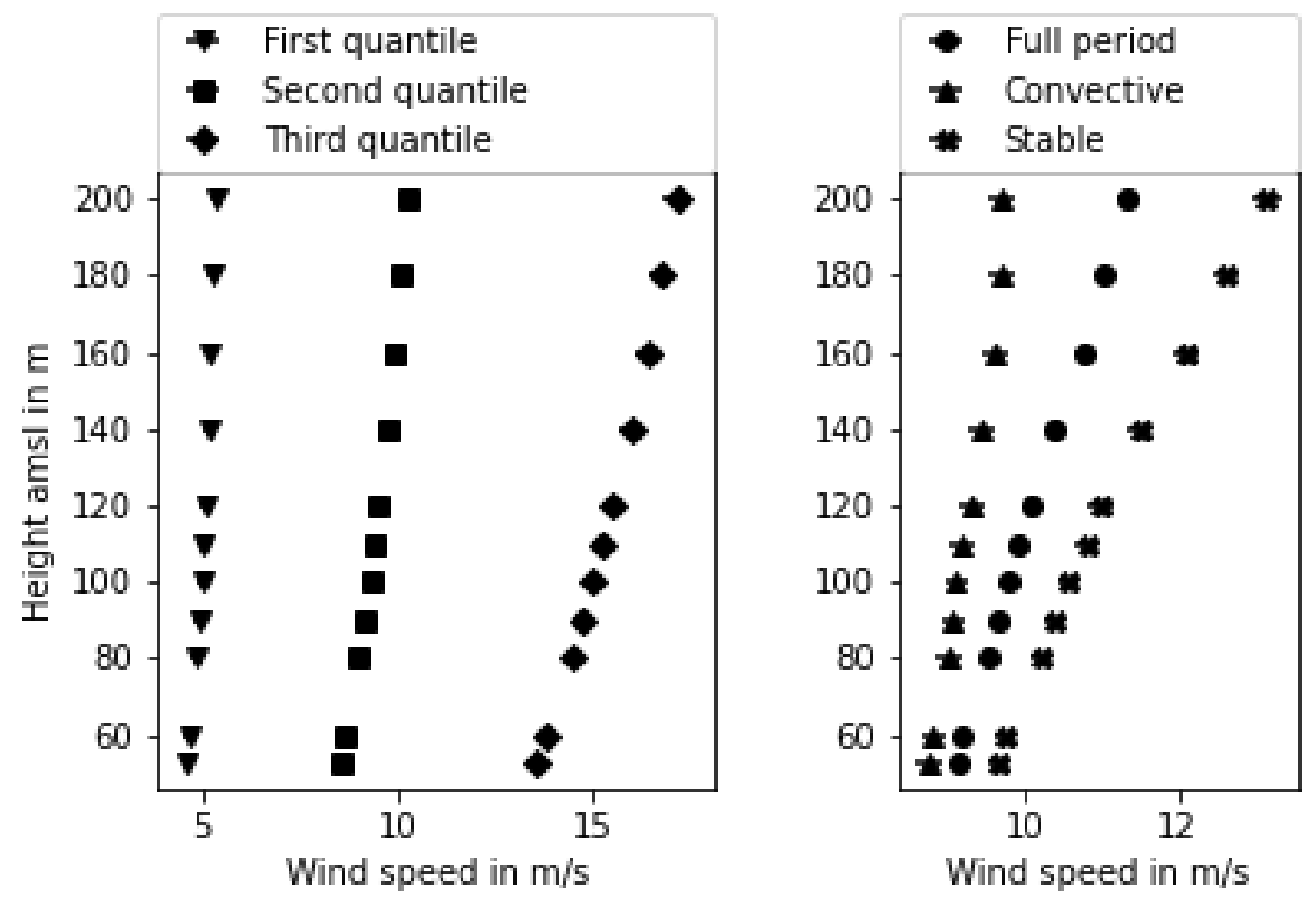

Figure B-6: Mean wind speed profiles based on the height averaged wind speed distribution and stability conditions. The distribution is divided into three quantiles with equal number of data points in each quantiles (33.33 percentile bins). Error bars are standard errors of mean estimated by method described in Ch:3.4.1 

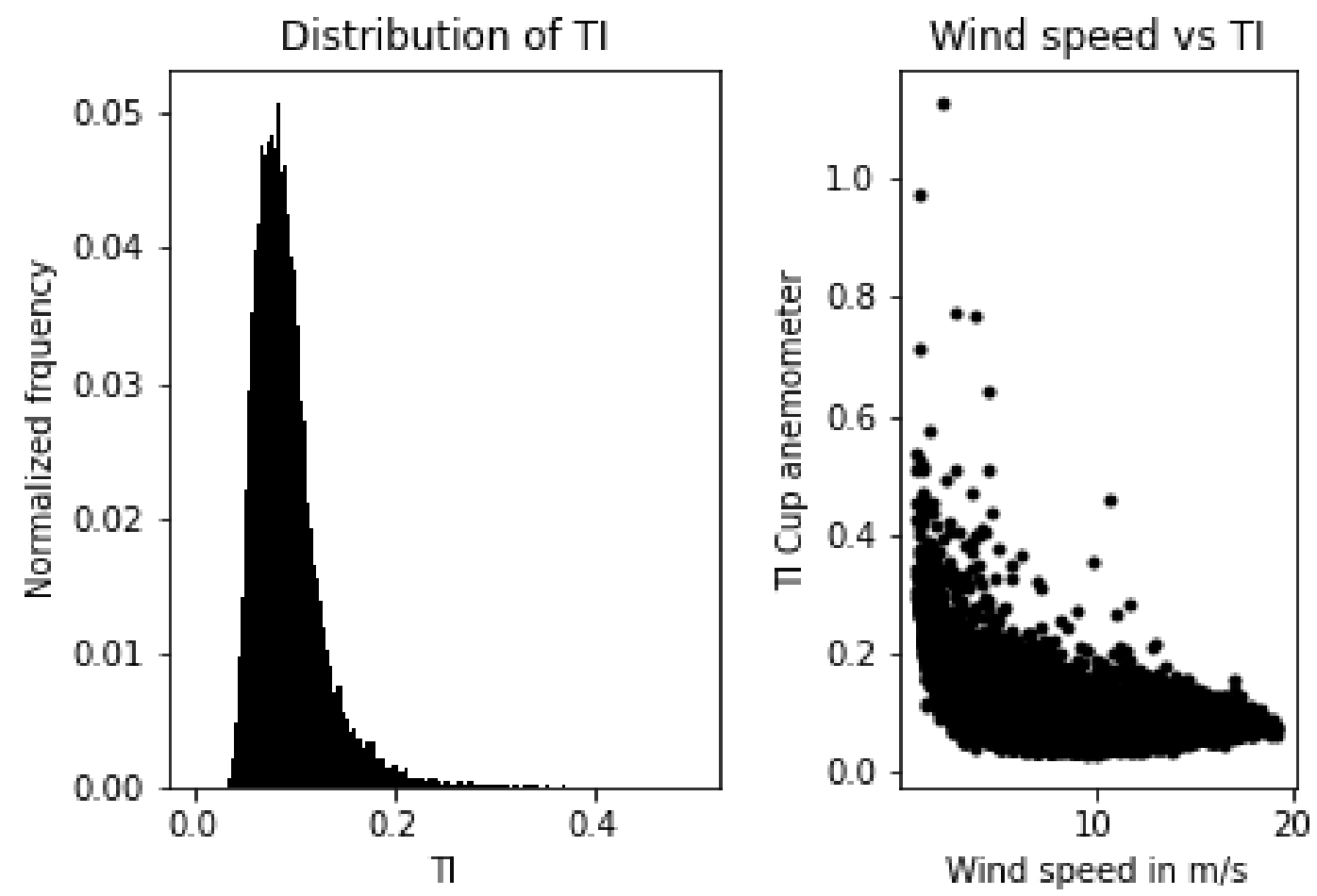

Figure B-7: Turbulence intensity distribution and comparison to mean wind speed measured by cup anemometer. 


\section{TI- Industry vs Met convention}
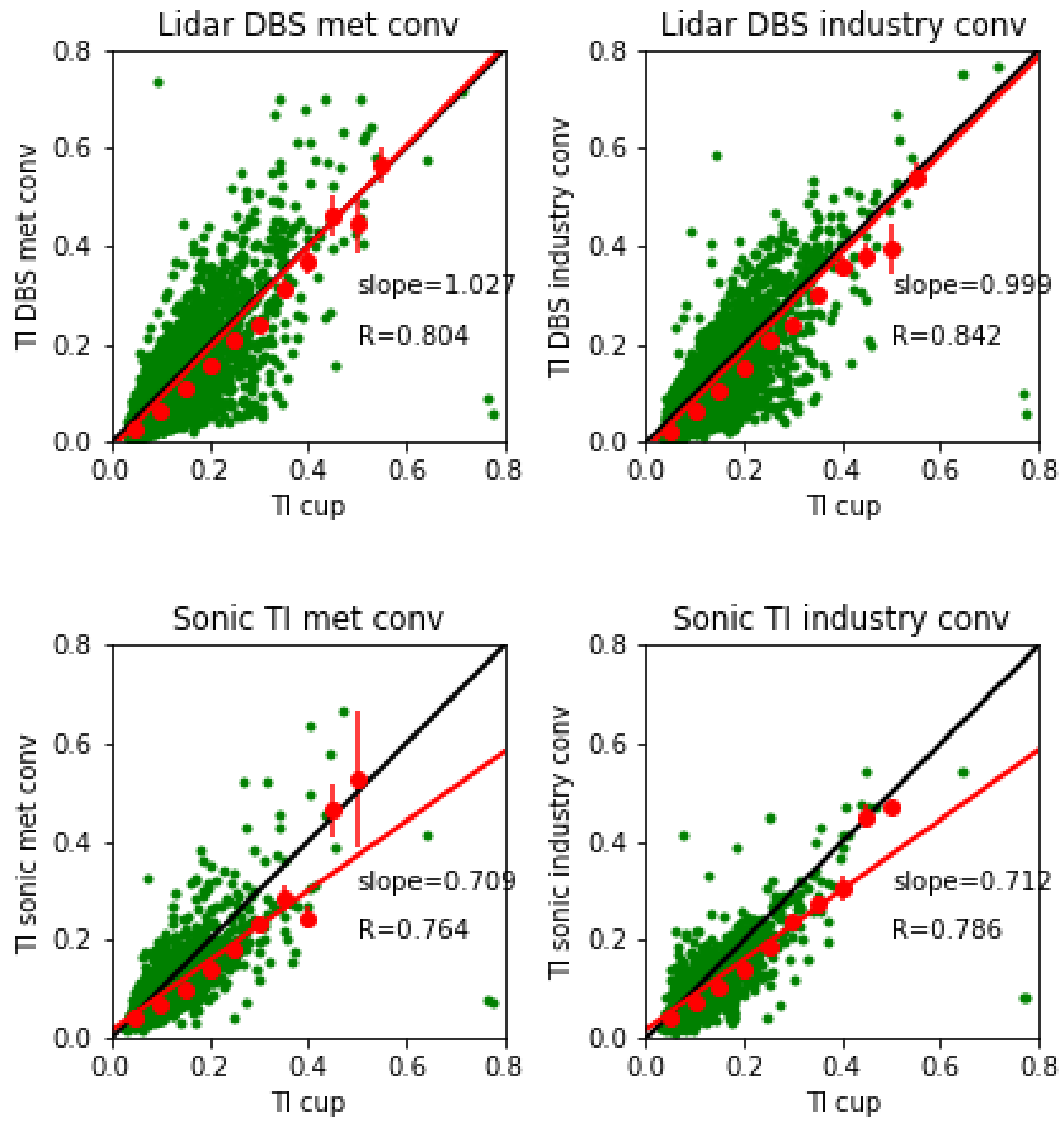

Figure B-8: Comparison of TI using Meteorological (left panels) and Industry convention (right) to calculate TI for WindCube v2.0 lidar at $53 \mathrm{~m}$ amsl (top) and sonic anemometer at $20 \mathrm{~m}$ amsl (bottom) with cup anemometer at $26 \mathrm{~m}$ amsl. The black line shows the slope 1 line and red line shows the linear regression fit, slope and the Pearson correlation coefficient are displayed on the panel. The red markers with error bars are bin averaged TI. 


\section{$\mathrm{Tl}$ comparison with cup}
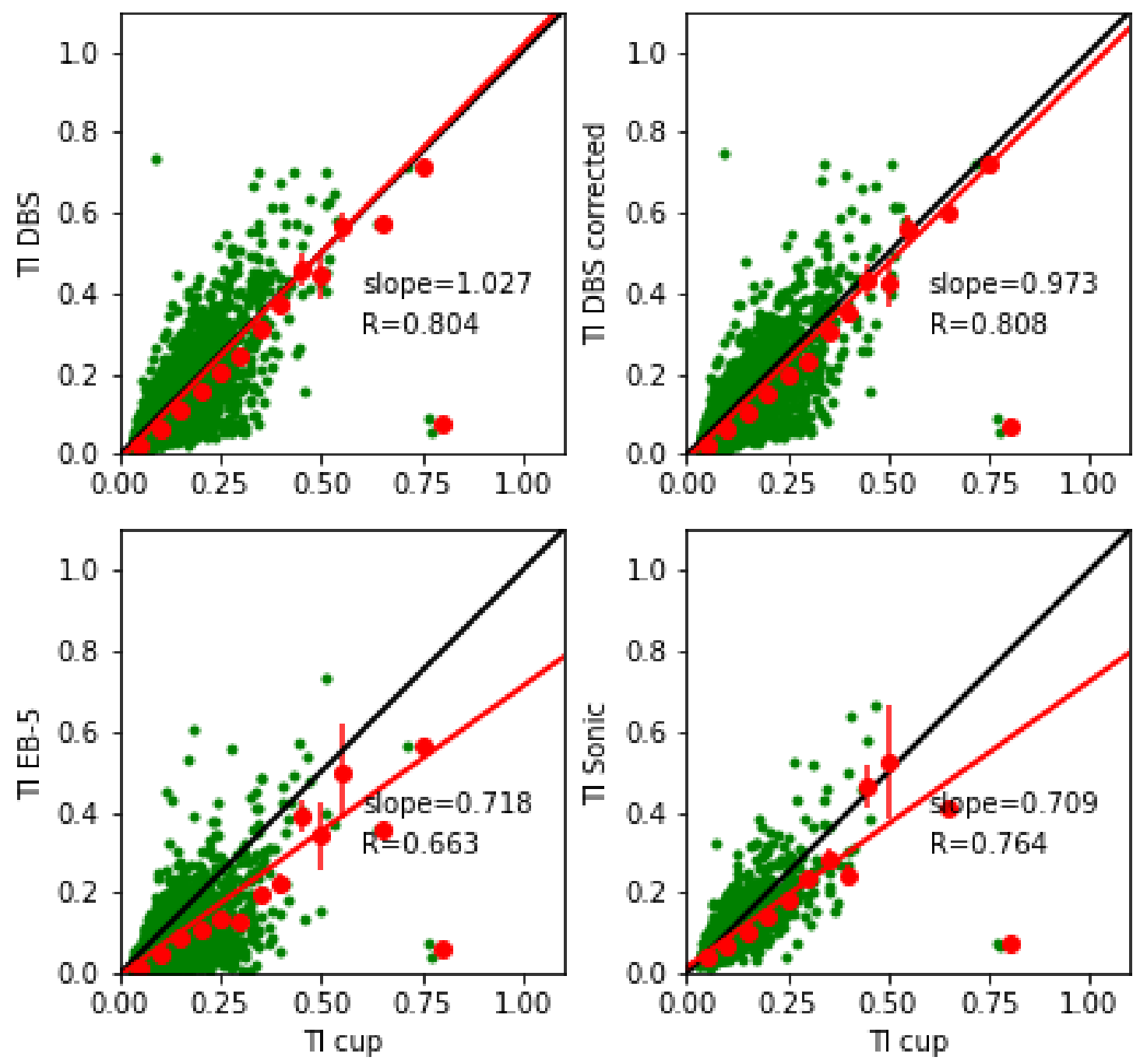

Figure B-9: Comparison of TI for the full period for: DBS, DBS corrected and EB5 methods for WindCube v2.0 lidar at $53 \mathrm{~m}$ and sonic anemometer at $20 \mathrm{~m}$ (Sonic data available only from late February onwards) with cup anemometer at $26 \mathrm{~m}$ amsl. Statistics displayed on the figure are same as in (Fig: B-8). 

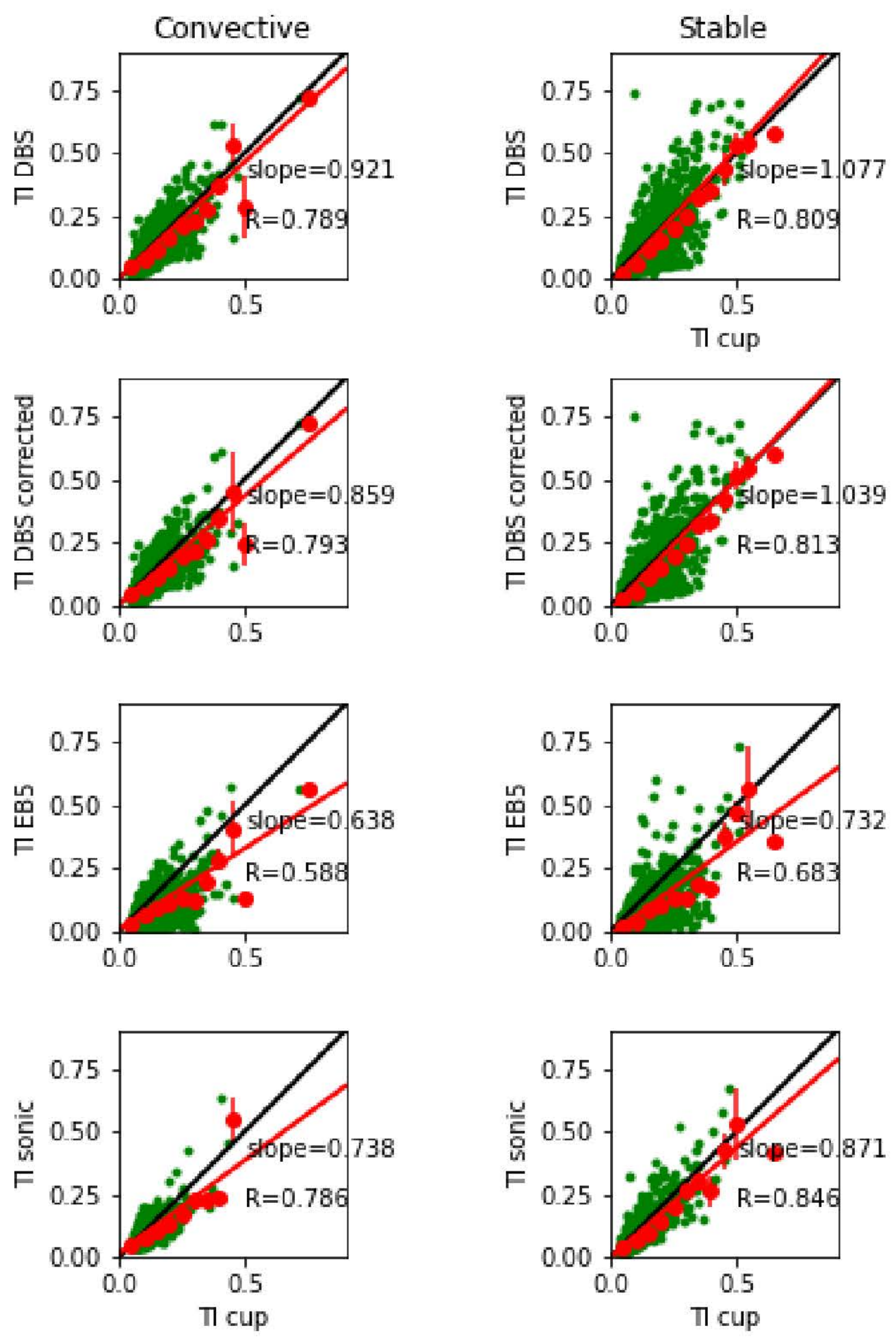

Figure B-10: Comparison of TI during convective (left) and stable (right) for (top to bottom): DBS method, DBS corrected method, EB-5 method and sonic anemometer (Sonic data available only from late February onwards) with cup anemometer at 26 $\mathrm{m}$ amsl. Statistics displayed on the figure are same as in (Fig: B-8). 

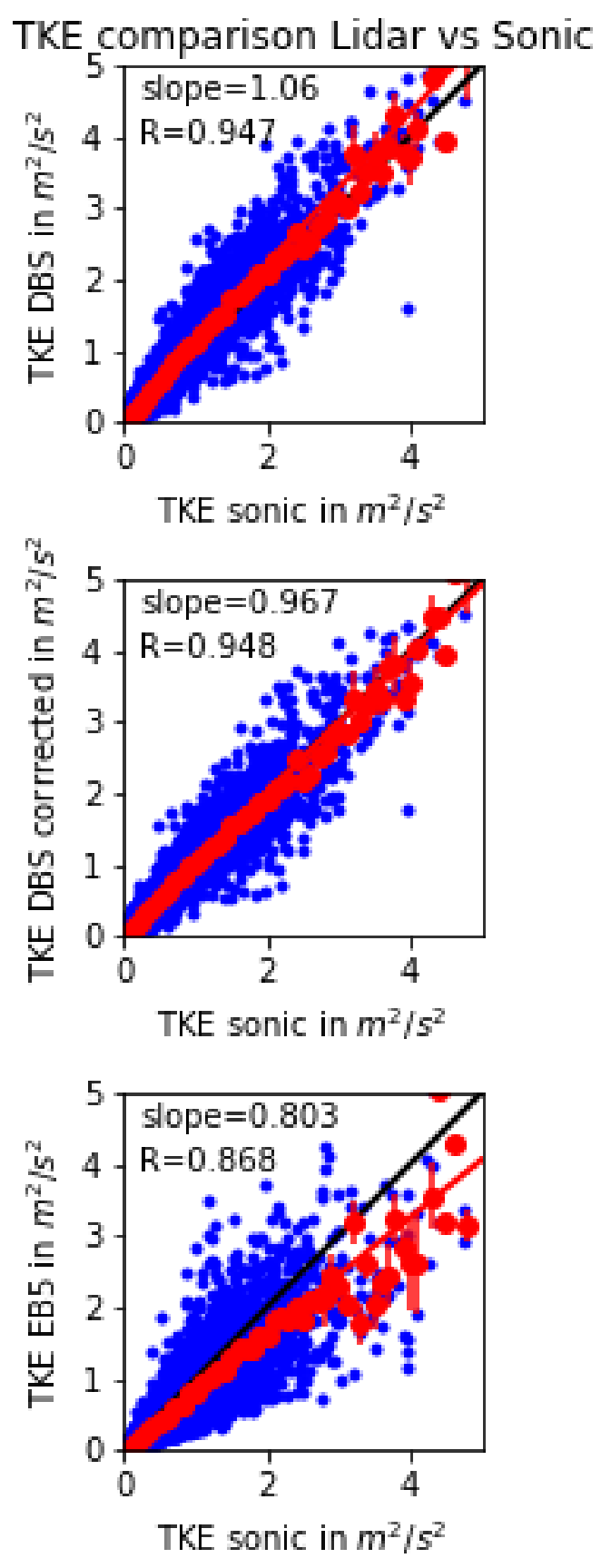

Figure B-11: TKE comparison of (top to bottom): DBS, DBS corrected and EB-5 method for WindCube v2.0 lidar at $53 \mathrm{~m}$ amsl with sonic anemometer at $20 \mathrm{~m}$ amsl from late February onwards. The black line shows the slope 1 line and red line shows the linear regression line, slope. The Pearson correlation coefficient are displayed on the panel. The red markers with error bars are bin averaged TKE. Error bars are standard error of mean computed as described in Sec. 3.4.1 

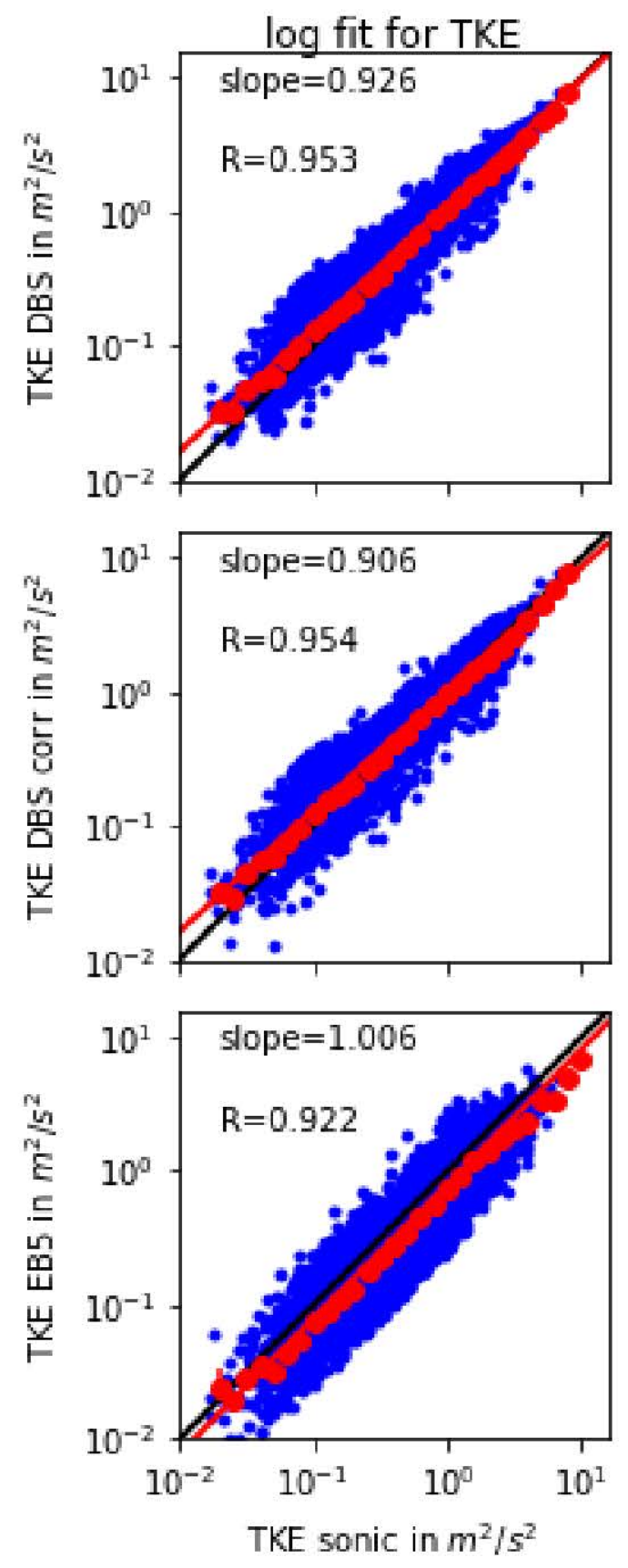

Figure B-12: log-TKE comparison of the three methods for lidar at $53 \mathrm{~m}$ amsl with TKE measured by sonic anemometer at $20 \mathrm{~m}$ amsl from late February onwards. Statistics displayed on figure are same as in (Fig: B-11).The slope and Pearson correlation coefficient for log-TKE linear regression fit is displayed. 

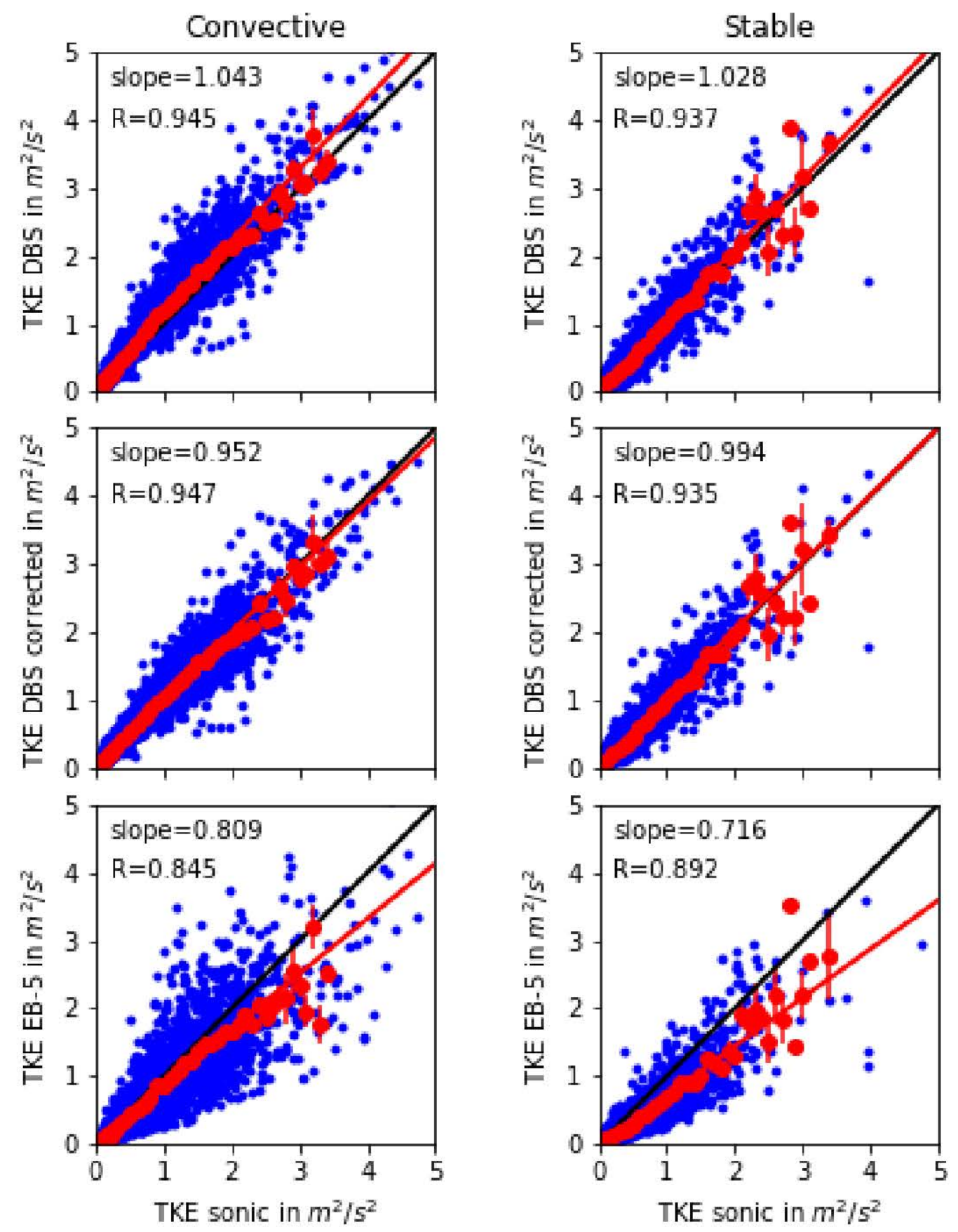

Figure B-13: TKE comparison during convecive (left) and stable (right) of (top to bottom): DBS, DBS corrected and EB-5 method for WindCube v2.0 lidar at $53 \mathrm{~m}$ amsl with sonic anemometer at $20 \mathrm{~m}$ amsl from late February onwards. Statistics displayed on figure are same as in B-11 

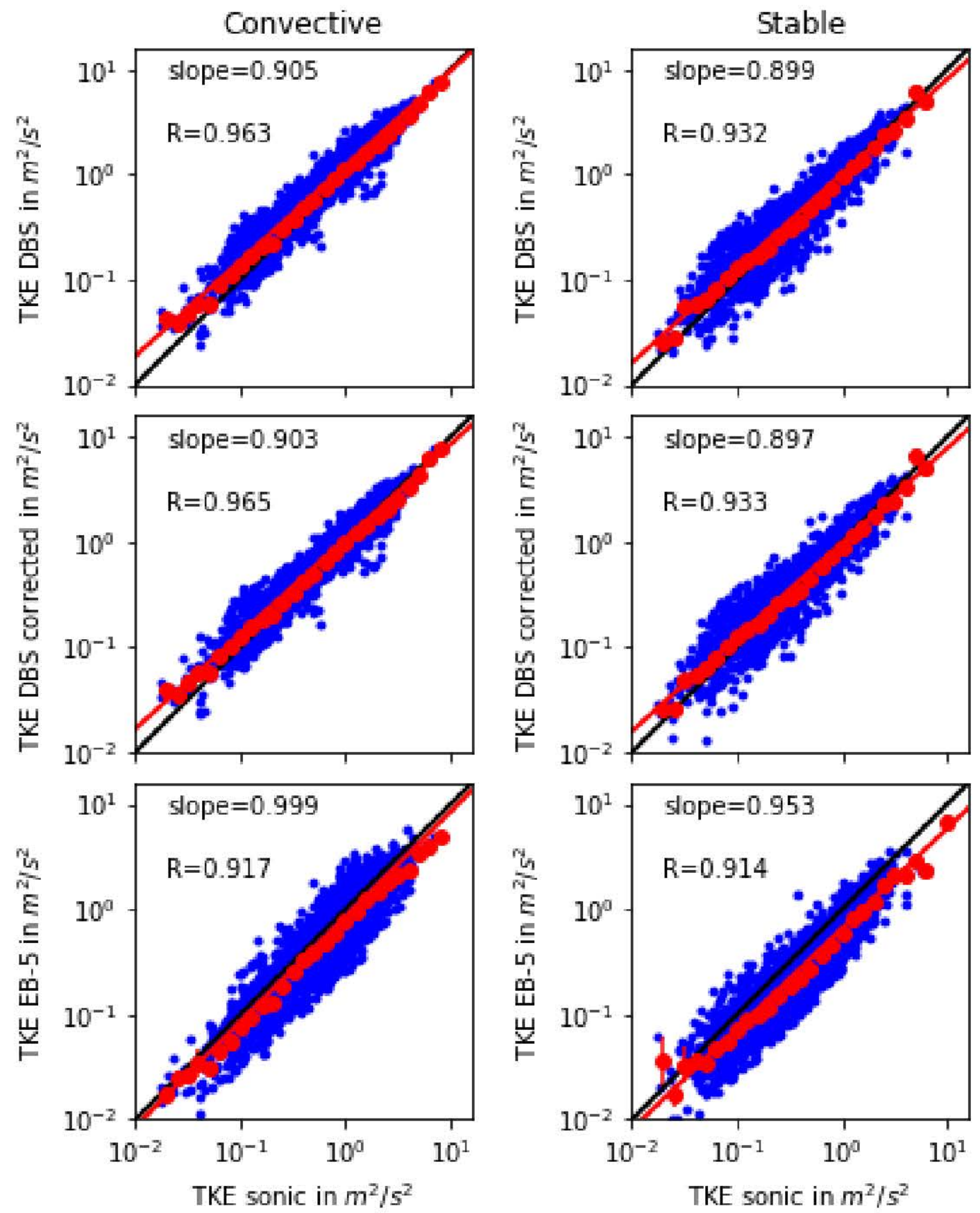

Figure B-14: log-TKE comparison during convecive (left) and stable (right) of (top to bottom): DBS, DBS corrected and EB-5 method for WindCube v2.0 lidar at 53 $\mathrm{m}$ amsl with sonic anemometer at $20 \mathrm{~m}$ amsl from late February onwards. Statistics displayed on figure are same as in (Fig: B-11). The slope and Pearson correlation coefficient for log-TKE linear regression fit is displayed. 

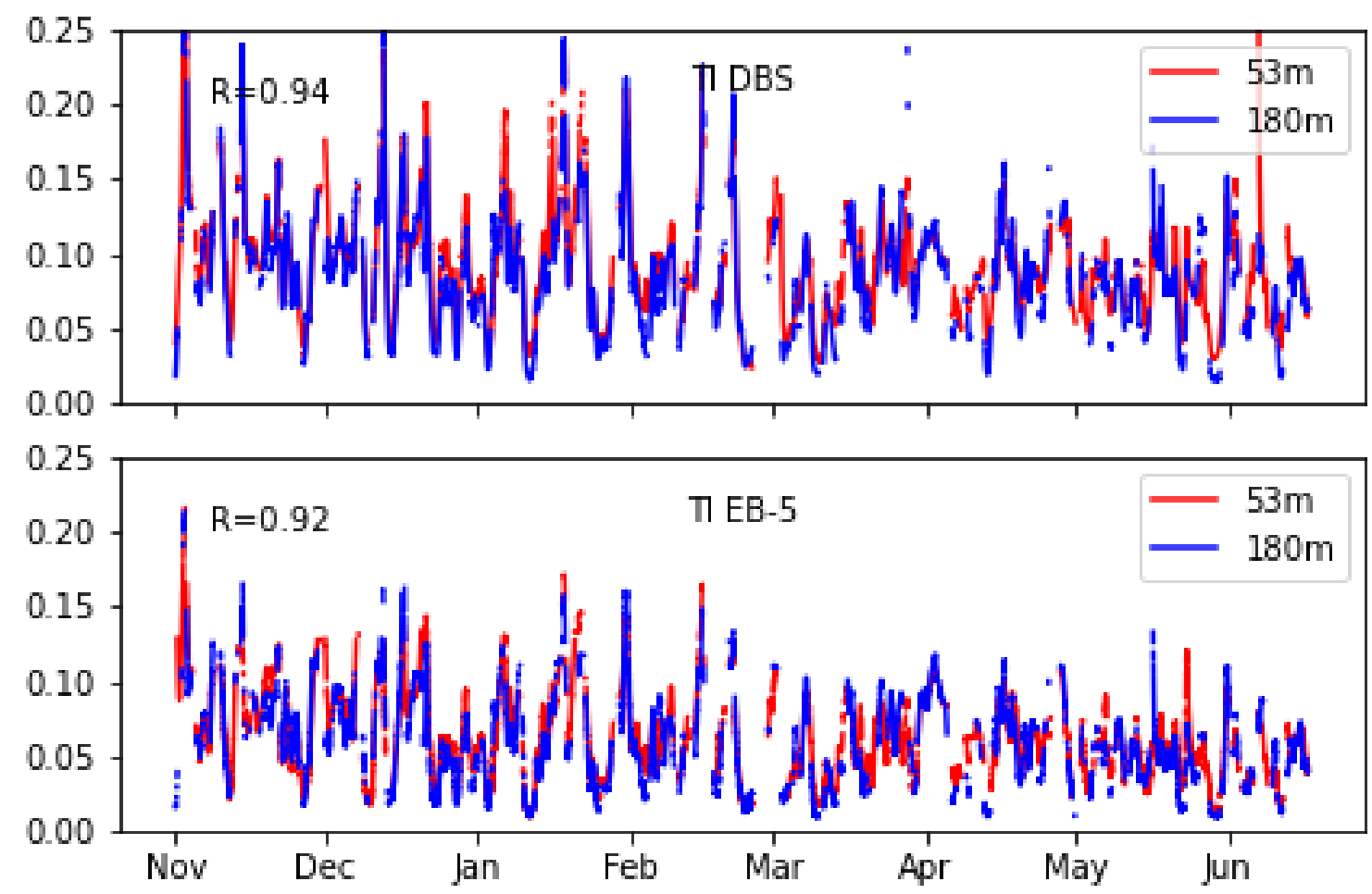

Figure B-15: 40-hour low pass filtered TI time series for DBS and EB-5 method for WindCube v2.0 lidar at $53 \mathrm{~m}$ and $180 \mathrm{~m}$ amsl. The Pearson correlation coefficient between time series at the two heights is displayed on the panel. 
TI DBS profile for stability conditions

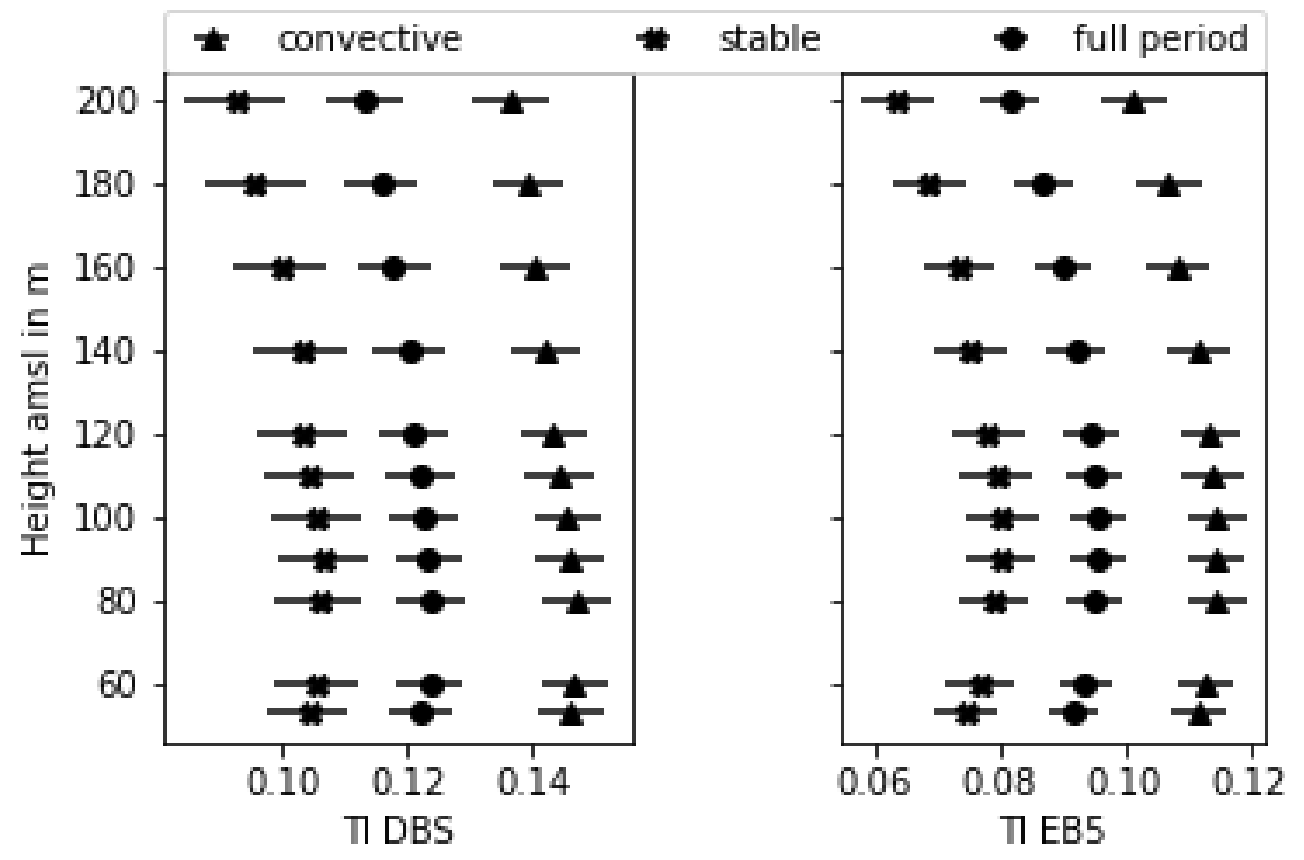

Figure B-16: Mean TI profile for DBS (left) and EB-5 (right) during convective and stable conditions and for the full study period. 

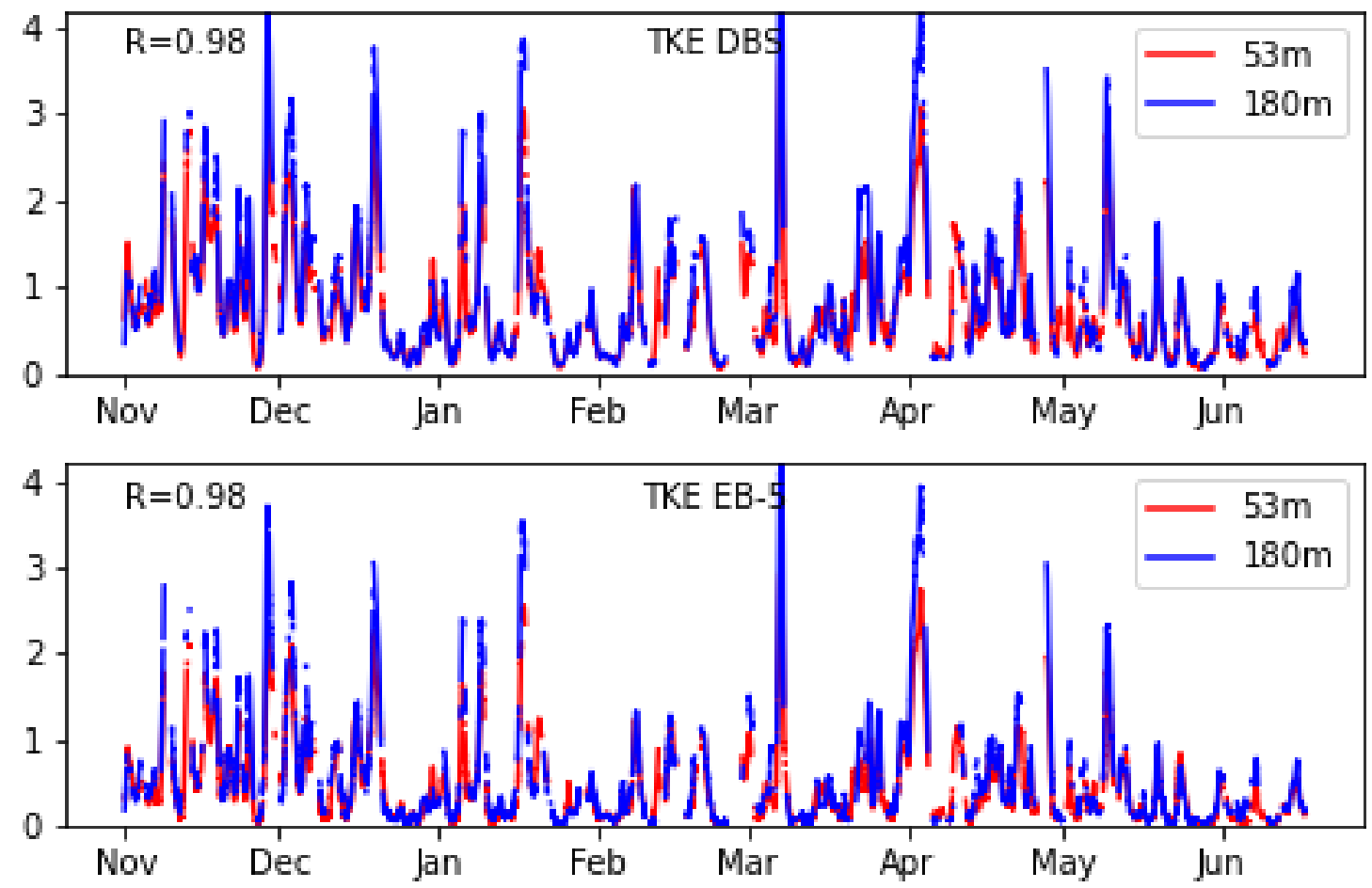

Figure B-17: 40-hour low pass filtered TKE time series for DBS and EB-5 method for WindCube v2.0 lidar at $53 \mathrm{~m}$ and $180 \mathrm{~m}$ amsl. The Pearson correlation coefficient between time series at the two heights is displayed on the panel. 


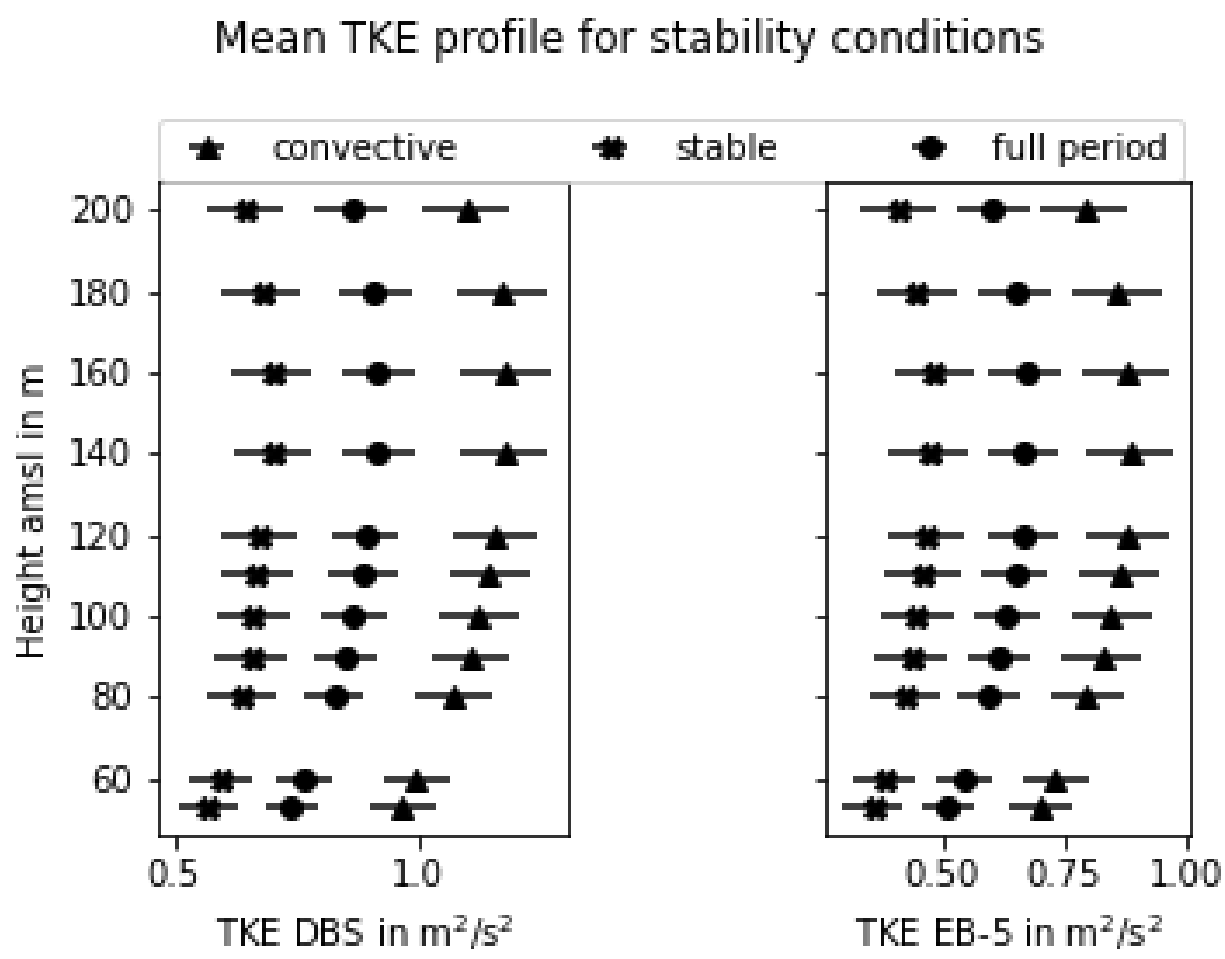

Figure B-18: Mean TKE profile for DBS (left) and EB-5 (right) during convective and stable conditions and for the full study period. 
TKE DBS for convective and stable conditions

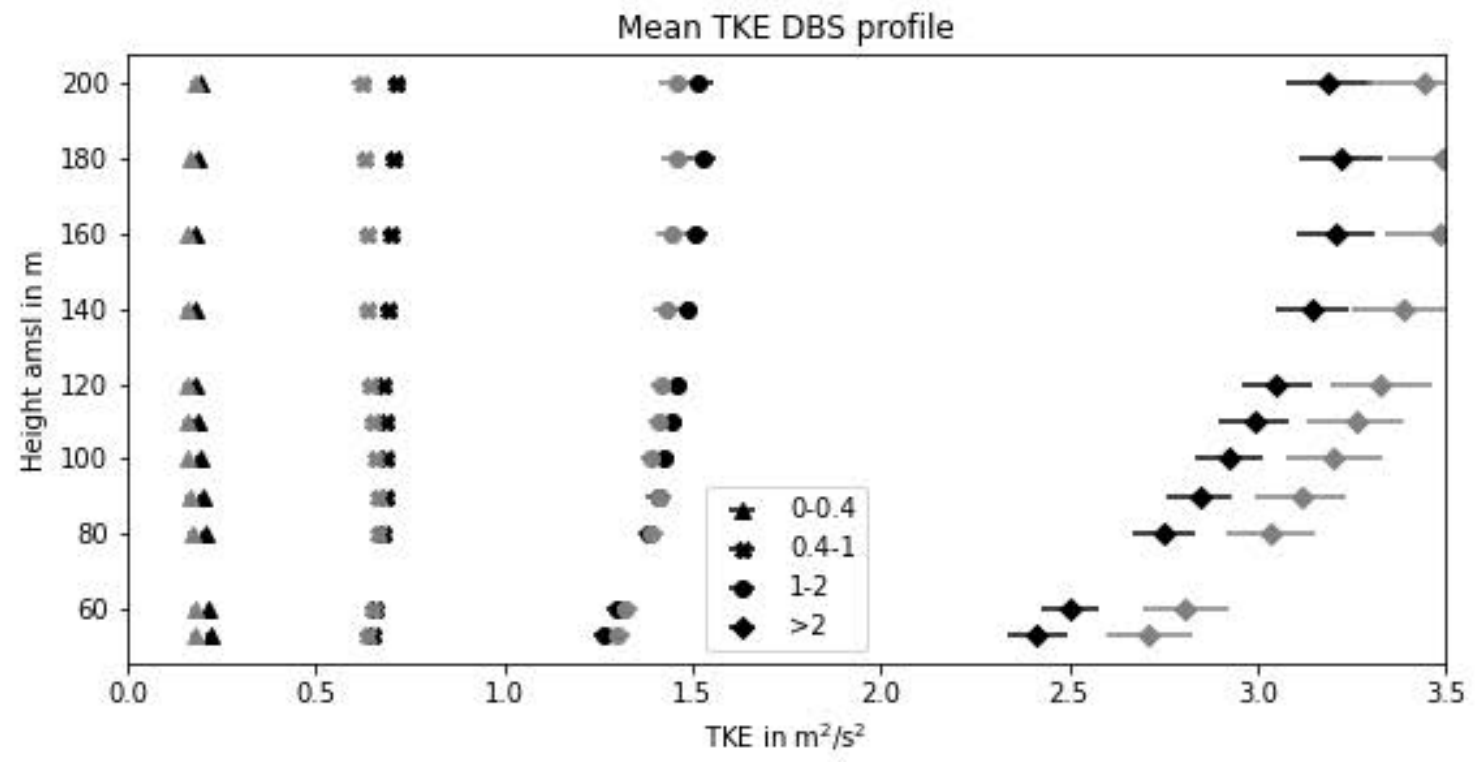

Height averaged TKE distribution

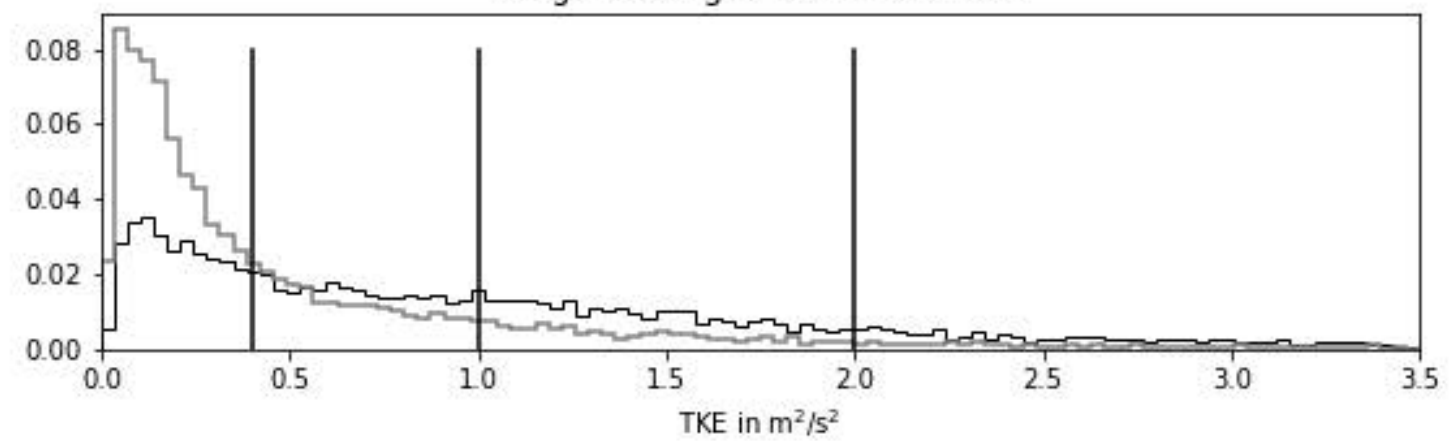

Figure B-19: Top: Mean TKE profile for four bins (shown in bottom panel) for stable (grey) and convective (black). Bottom: Height averaged TKE distribution using DBS method for stable (grey) and convective (black). The bins are divided as $(0,0.4)(0.4$, 1) $(1,2)(>2)$ and shown with vertical lines. The standard error of mean is estimated by method described in Sec.3.4.1. 


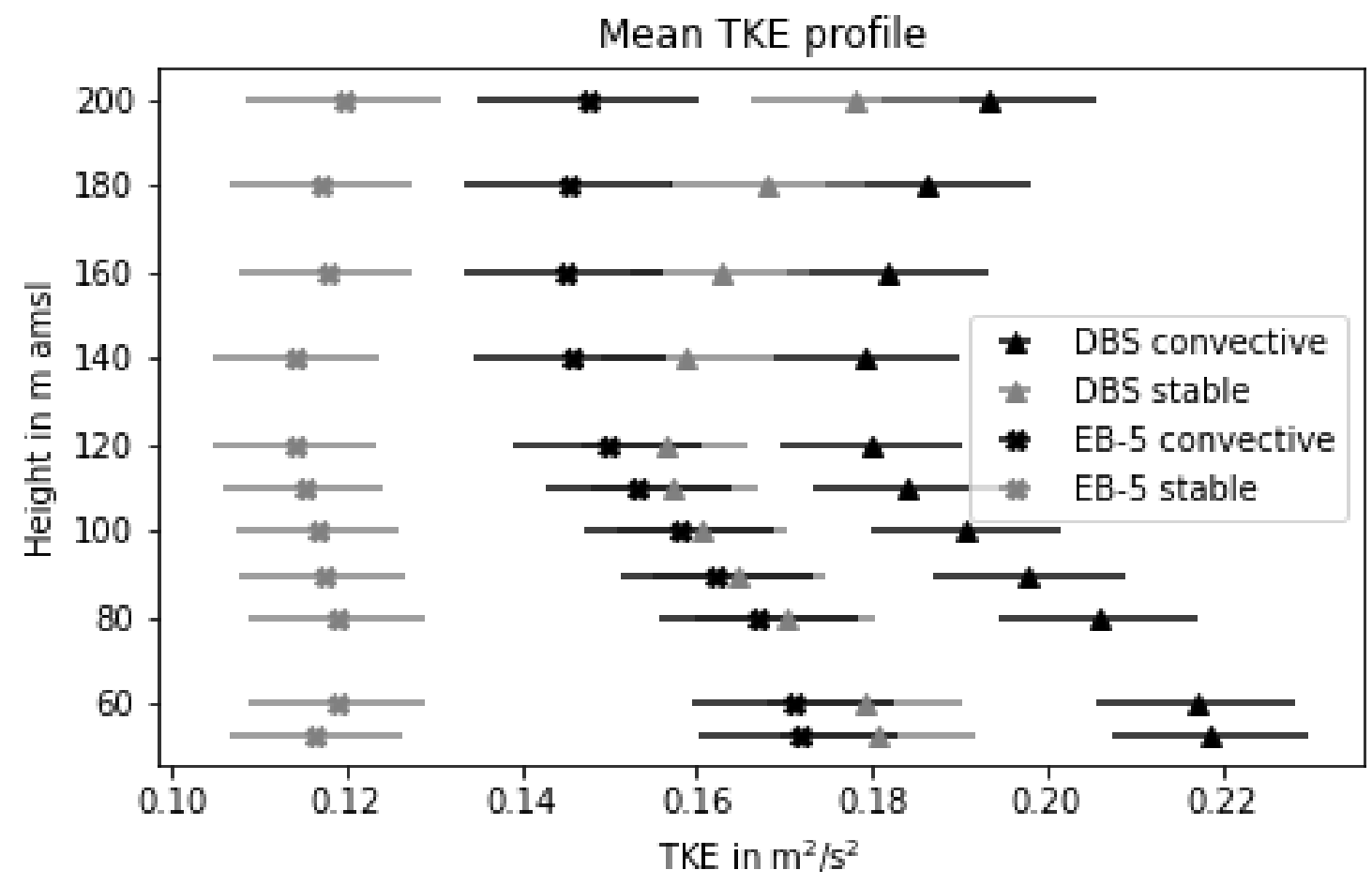

Figure B-20: Mean TKE profile for the first bin (TKE 0 to $0.4 \mathrm{~m}^{2} / \mathrm{s}^{2}$ ) in Figure: B-19 for DBS (triangles) and EB-5 (cross) during convective (black) and stable (grey) conditions. The standard error of mean is estimated by method described in Sec.3.4.1. 


\section{Bibliography}

[1] Janice L Coen, Marques Cameron, John Michalakes, Edward G Patton, Philip J Riggan, and Kara M Yedinak. Wrf-fire: coupled weather-wildland fire modeling with the weather research and forecasting model. Journal of Applied Meteorology and Climatology, 52(1):16-38, 2013.

[2] Michael Frech. Estimating the turbulent energy dissipation rate in an airport environment. Boundary-layer meteorology, 123(3):385-393, 2007.

[3] Nicola Bodini, Julie K. Lundquist, and Anthony Kirincich. U.S. East Coast Lidar Measurements Show Offshore Wind Turbines Will Encounter Very Low Atmospheric Turbulence. Geophysical Research Letters, 46(10):5582-5591, 2019.

[4] Ewan J. O'connor, Anthony J. Illingworth, Ian M. Brooks, Christopher D. Westbrook, Robin J. Hogan, Fay Davies, Brooks, and Barbara J. A method for estimating the turbulent kinetic energy dissipation rate from a vertically pointing doppler lidar, and independent evaluation from balloon-borne in situ measurements. Journal of Atmospheric and Oceanic Technology, 27(10):1652-1664, 2010.

[5] Michael E. Rhodes and Julie K. Lundquist. The Effect of Wind-Turbine Wakes on Summertime US Midwest Atmospheric Wind Profiles as Observed with GroundBased Doppler Lidar. Boundary-Layer Meteorology, 149(1):85-103, 2013.

[6] Nicola Bodini, Julie K. Lundquist, Raghavendra Krishnamurthy, Mikhail Pekour, Larry K. Berg, and Aditya Choukulkar. Spatial and temporal variability of turbulence dissipation rate in complex terrain. Atmospheric Chemistry and Physics, 19(7):4367-4382, 2019.

[7] Ben Yang, Yun Qian, Larry K. Berg, Po Lun Ma, Sonia Wharton, Vera Bulaevskaya, Huiping Yan, Zhangshuan Hou, and William J. Shaw. Sensitivity of Turbine-Height Wind Speeds to Parameters in Planetary Boundary-Layer and Surface-Layer Schemes in the Weather Research and Forecasting Model. Boundary-Layer Meteorology, 162(1):117-142, 2017.

[8] Larry K. Berg, Ying Liu, Ben Yang, Yun Qian, Joseph Olson, Mikhail Pekour, Po Lun Ma, and Zhangshuan Hou. Sensitivity of Turbine-Height Wind Speeds to Parameters in the Planetary Boundary-Layer Parametrization Used in the Weather Research and Forecasting Model: Extension to Wintertime Conditions. Boundary-Layer Meteorology, 170(3):507-518, 2019. 
[9] Rozenn Wagner, B Cañadillas, A Clifton, S Feeney, N Nygaard, M Poodt, C St Martin, E Tüxen, and JW Wagenaar. Rotor equivalent wind speed for power curve measurement-comparative exercise for iea wind annex 32. In Journal of Physics: Conference Series, volume 524, page 012108. IOP Publishing, 2014.

[10] Sonia Wharton and Julie K Lundquist. Sphere anemometer-a faster alternative solution to cup anemometry Recent citations Atmospheric stability affects wind turbine power collection. Journal of Physics: Conference Series OPEN ACCESS.

[11] Matthew J Churchfield, Sang Lee, John Michalakes, and Patrick J Moriarty. A numerical study of the effects of atmospheric and wake turbulence on wind turbine dynamics. Journal of turbulence, (13):N14, 2012.

[12] Nikolay Dimitrov, Anand Natarajan, and Jakob Mann. Effects of normal and extreme turbulence spectral parameters on wind turbine loads. Renewable Energy, 101:1180-1193, 2017.

[13] A. Sathe, J. Mann, T. Barlas, W. A.A.M. Bierbooms, and G. J.W. Van Bussel. Influence of atmospheric stability on wind turbine loads. Wind Energy, 16(7):1013-1032, 102013.

[14] John Dalsgaard Sørensen, S Frandsen, and NJ Tarp-Johansen. Effective turbulence models and fatigue reliability in wind farms. Probabilistic Engineering Mechanics, 23(4):531-538, 2008.

[15] Wout Weijtens, Nymfa Noppe, Tim Verbelen, Alexandros Iliopoulos, and Christof Devriendt. Offshore wind turbine foundation monitoring, extrapolating fatigue measurements from fleet leaders to the entire wind farm. In Journal of Physics: Conference Series, volume 753, page 092018, 2016.

[16] Christiane Adcock and Ryan N King. Data-driven wind farm optimization incorporating effects of turbulence intensity. In 2018 Annual American Control Conference $(A C C)$, pages 695-700. IEEE, 2018.

[17] Rod Frehlich. Scanning doppler lidar for input into short-term wind power forecasts. Journal of Atmospheric and Oceanic Technology, 30(2):230-244, 2013.

[18] Valerie M. Kumer, Joachim Reuder, Manfred Dorninger, Rudolf Zauner, and Vanda Grubišić. Turbulent kinetic energy estimates from profiling wind LiDAR measurements and their potential for wind energy applications. Renewable Energy, 99:898-910, 2016.

[19] Sonia Wharton and Julie K. Lundquist. Assessing atmospheric stability and its impacts on rotor-disk wind characteristics at an onshore wind farm, 2012.

[20] IEC. Iec 61400-12-1. International Electrotechnical Commission, 2005:179, 2005. 
[21] Alfredo Peña, Charlotte Bay Hasager, Julia Lange, Jan Anger, Merete Badger, Ferhat Bingöl, Oliver Bischoff, Jean-Pierre Cariou, Fiona Dunne, Stefan Emeis, et al. Remote sensing for wind energy. DTU Wind Energy, 2013.

[22] Alfredo Pena, Charlotte B Hasager Eds, Oliver Bischoff, Sten T Frandsen, Jakob Mann, and Juan Jose Trujillo. Remote Sensing for Wind Energy Wind Energy, volume 3068. 2010.

[23] A. Sathe, R. M. Banta, L. Pauscher, K. Vogstad, D. Schlipf, and S. Wylie. Estimating Turbulence Statistics and Parameters from International Energy Agency Expert Report Expert Report IEA Task 32. Number October. 2015.

[24] Sonia Wharton and Julie K. Lundquist. Atmospheric stability affects wind turbine power collection. Environmental Research Letters, 7(1), 2012.

[25] Julia Gottschall, Brian Gribben, Detlef Stein, and Ines Würth. Floating lidar as an advanced offshore wind speed measurement technique: Current technology status and gap analysis in regard to full maturity. Wiley Interdisciplinary Reviews: Energy and Environment, 6(5):e250, 2017.

[26] A. Sathe and J. Mann. A review of turbulence measurements using ground-based wind lidars. Atmospheric Measurement Techniques, 6(11):3147-3167, 2013.

[27] A. Morales, M. Wächter, and J. Peinke. Characterization of wind turbulence by higher-order statistics. Wind Energy, 15(3):391-406, 42012.

[28] Michael Courtney, Rozenn Wagner, and Petter Lindelöw. Commercial lidar profilers for wind energy: A comparative guide. In Proc. European Wind Energy Conference, Brussels, Belgium, 2008.

[29] A. Sathe, J. Mann, J. Gottschall, and M. S. Courtney. Can wind lidars measure turbulence? Journal of Atmospheric and Oceanic Technology, 28(7):853-868, 2011.

[30] R G Strauch, DA Merritt, KP Moran, KB Earnshaw, and D Van De Kamp. The colorado wind-profiling network. Journal of Atmospheric and Oceanic Technology, 1(1):37-49, 1984.

[31] C Sak, R Liu, DS-K Ting, and GW Rankin. The role of turbulence length scale and turbulence intensity on forced convection from a heated horizontal circular cylinder. Experimental thermal and fluid science, 31(4):279-289, 2007.

[32] RH Shaw, G Den Hartog, KM King, and GW Thurtell. Measurements of mean wind flow and three-dimensional turbulence intensity within a mature corn canopy. Agricultural Meteorology, 13(3):419-425, 1974.

[33] PW Chan. Measurement of turbulence intensity profile by a mini-sodar. Meteorological Applications: A journal of forecasting, practical applications, training techniques and modelling, 15(2):249-258, 2008. 
[34] Roymond S Hunter, B Maribo Pedersen, TF Pedersen, H Klug, N van der Borg, N Kelley, and JA Dahlberg. Recommended practices for wind turbine testing and evaluation. 11. wind speed measurement and use of cup anemometry. 1. IEA, 1999.

[35] M Hölling, B Schulte, S Barth, and J Peinke. Sphere anemometer - a faster alternative solution to cup anemometry. Journal of Physics: Conference Series, 75:012064, 72007.

[36] Using HPC for Computational Fluid Dynamics. Elsevier, 2015.

[37] JC Wyngaard. Turbulence in the Atmosphere. 2010.

[38] Ferhat Bingöl, Jakob Mann, and Dimitri Foussekis. Conically scanning lidar error in complex terrain. Meteorologische Zeitschrift, 18(2):189-195, 2009.

[39] Wynn L Eberhard, Richard E Cupp, and Kathleen R Healy. Doppler Lidar Measurement of Profiles of Turbulence and Momentum Flux. Journal of Atmospheric and Oceanic Technology, 6(5):809-819.

[40] Tzvi Gal-Chen, Mei Xu, and W. L. Eberhard. Estimations of atmospheric boundary layer fluxes and other turbulence parameters from Doppler lidar data. Journal of Geophysical Research, 97(D17):409-423, 1992.

[41] A. Sathe, J. Mann, N. Vasiljevic, and G. Lea. A six-beam method to measure turbulence statistics using ground-based wind lidars. Atmospheric Measurement Techniques, 8(2):729-740, 2015.

[42] Jennifer F. Newman, Petra M. Klein, Sonia Wharton, Ameya Sathe, Timothy A. Bonin, Phillip B. Chilson, and Andreas Muschinski. Evaluation of three lidar scanning strategies for turbulence measurements. Atmospheric Measurement Techniques, 9(5):1993-2013, 2016.

[43] KA Browning and $\mathrm{R}$ Wexler. The determination of kinematic properties of a wind field using doppler radar. Journal of Applied meteorology and climatology, 7(1):105-113, 1968.

[44] Ferhat Bingöl, Jakob Mann, and Dimitri Foussekis. Lidar error estimation with wasp engineering. In IOP Conference Series: Earth and Environmental Science, volume 1, page 012058. IOP Publishing, 2008.

[45] J Newman, T Bonin, P Klein, and S Wharton. Optimizing Lidar Scanning Strategies for Wind Energy Turbulence Measurements. 21st Symposium on Boundary Layers and Turbulence, 2013.

[46] Rod Frehlich. Estimation of velocity error for doppler lidar measurements. Journal of Atmospheric and Oceanic Technology, 18(10):1628-1639, 2001. 
[47] Timothy A. Bonin, Aditya Choukulkar, W. Alan Brewer, Scott P. Sandberg, Ann M. Weickmann, Yelena L. Pichugina, Robert M. Banta, Steven P. Oncley, and Daniel E. Wolfe. Evaluation of turbulence measurement techniques from a single Doppler lidar. Atmospheric Measurement Techniques, 10(8):3021-3039, 2017.

[48] Anthony Kirincich. A Metocean Reference Station for Offshore Wind Energy Research in the U.S. Journal of Physics: Conference Series, 1452(1), 2020.

[49] Turbulence intensity - Glossary of Meteorology.

[50] Nicola Bodini, Julie K. Lundquist, and Mike Optis. Can machine learning improve the model representation of turbulent kinetic energy dissipation rate in the boundary layer for complex terrain? Geoscientific Model Development, 13(9):4271-4285, 2020.

[51] Ameya Sathe and Jakob Mann. Measurement of turbulence spectra using scanning pulsed wind lidars. Journal of Geophysical Research: Atmospheres, 117(D1), 2012.

[52] Jielun Sun, Larry Mahrt, Robert M. Banta, and Yelena L. Pichugina. Turbulence regimes and turbulence intermittency in the stable boundary layer: During CASES-99. Journal of the Atmospheric Sciences, 69(1):338-351, 2012. 